Université de Montréal

Suivi à long terme des effets d'un entrainement de la mémoire de travail (Cogmed) auprès d'enfants présentant un TDA/H

par Camille Rivard

Département de psychologie

Faculté des Arts et des Sciences

Essai doctoral présenté à la Faculté des Arts et des Sciences

en vue de l'obtention du grade de doctorat

en neuropsychologie clinique (D. Psy.)

Août 2017

(C) Camille Rivard, 2017 


\section{Résumé}

Le trouble déficitaire de l'attention avec ou sans hyperactivité (TDA/H) est fréquemment associé à un déficit de la mémoire de travail. De nouvelles interventions ciblant spécifiquement cette fonction ont ainsi été développées, dont le programme Cogmed. Or, les effets à long terme de ce type d'intervention demeurent peu étudiés et peu d'études tiennent compte de la présence de médication ou de troubles associés. Cette étude, en continuité avec Dentz et al. (soumis), vise à évaluer les effets à long terme du programme Cogmed comme adjuvant à la médication auprès de jeunes présentant un TDA/H mixte avec comorbidité(s) sous traitement pharmacologique. Trente-et-un participants âgés de 7 à 13 ans présentant un TDA/H associé à un trouble d'apprentissage, un trouble d'opposition avec provocation ou un syndrome de la Tourette ont pris part à une évaluation six mois post-intervention. Un design en double aveugle et un groupe contrôle actif ont été utilisés. Les résultats montrent que le programme Cogmed ne permet pas d'améliorer davantage la mémoire de travail du groupe expérimental, comparativement au groupe contrôle actif. Aucun effet de transfert différé n'est observé sur le fonctionnement cognitif de même que sur le rendement scolaire et les symptômes liés au TDA/H. La présente étude ne permet pas de démontrer l'efficacité, ni de recommander le programme Cogmed comme approche complémentaire à la médication auprès de la population ciblée. L'article qui découle de cette étude s'inscrit dans le cadre d'un projet d'essai doctoral et sera donc précédé d'un contexte théorique général sur le TDA/H et l'entrainement cognitif et suivi d'une conclusion présentant certaines pistes de réflexion.

Mots-clés : Entraînement cognitif, Mémoire de travail, Programme Cogmed, Maintien des acquis, Trouble déficitaire de l'attention/hyperactivité (TDAH), Neuropsychologie clinique 


\begin{abstract}
Background: Attention-deficit/hyperactivity disorder (ADHD) is a prevalent neurodevelopmental disorder associated with working memory deficits. In that optic, new interventions designed to train working memory have been developed. Cogmed Working Memory Training (CWMT) is a popular online training program that is said to increase working memory (WM) and related skills in the ADHD population. However, follow-up measures are not systematically included and long term effects of CWMT are controversial. Also, the number of studies examining transfer of learning and controlling for medication or comorbidities are limited. Objectives: This study, in continuity with Dentz et al. (submitted), investigates the long-term effects of CWMT combined with psychostimulant medication on WM and related skills. Methods: This study included 31 participants aged 7 to 13 years, diagnosed with ADHD (mixed subtype) associated with learning disorders, oppositional-defiant disorder and/or Tourette syndrome. Participants first completed CWMT and were evaluated at follow-up six months later. We used a randomized, double-blind, placebo-controlled design. Results: The results found no significant impact of CWMT at follow-up. There were no improvements of WM nor transfer effects on related skills, academic achievement and ADHD symptoms at six months' follow-up. Conclusion: Long-term efficacy of CWMT is not demonstrated. We can't support CWMT as an effective complementary treatment for children with ADHD and associated comorbidities. The article that will be submitted in the context of this doctoral essay is preceded by a literature review on ADHD and cognitive training and followed by a general conclusion offering future directions on CWMT.
\end{abstract}

Keywords: Attention Deficit Hyperactivity Disorder (ADHD), Working memory training, Cogmed program, Children, Clinical neuropsychology 


\section{Table des matières}

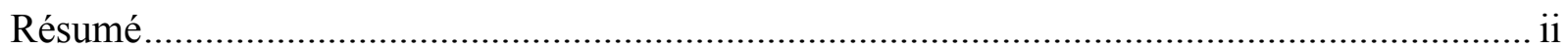

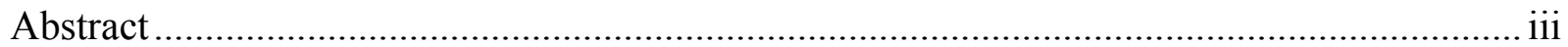

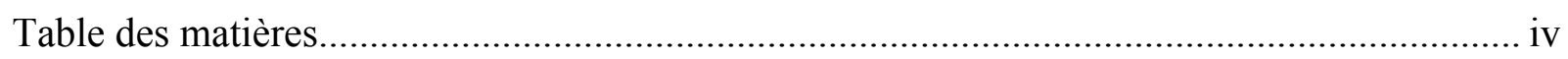

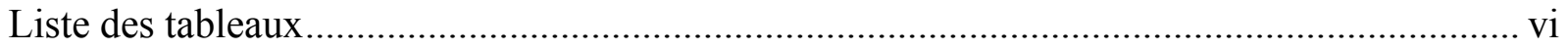

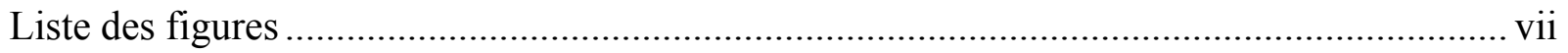

Liste des abréviations............................................................................................ viii

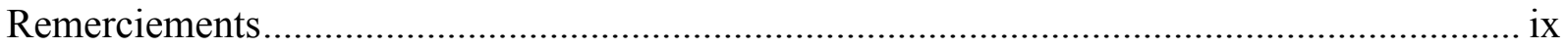

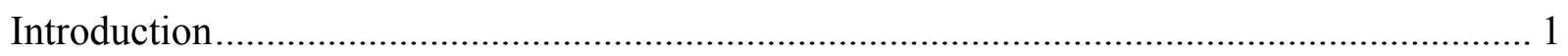

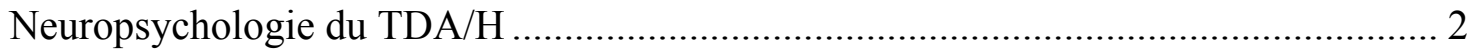

Traitement du TDA/H ..................................................................................... 5

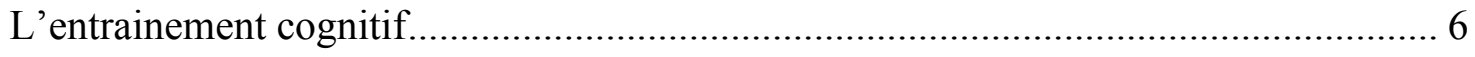

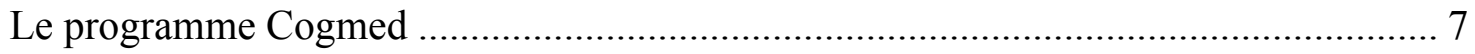

Efficacité du programme Cogmed ......................................................... 8

Article: Suivi à long terme des effets d'un entrainement de la mémoire de travail (Cogmed) auprès d'enfants présentant un TDA/H..................................................................... 14

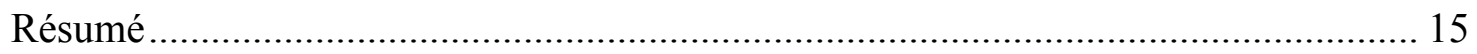

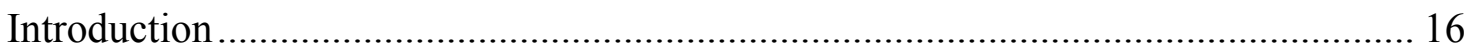

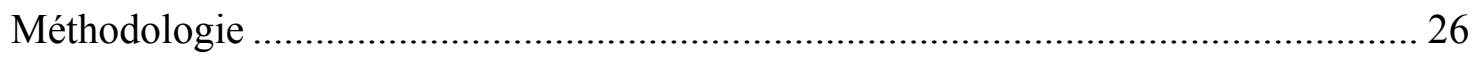

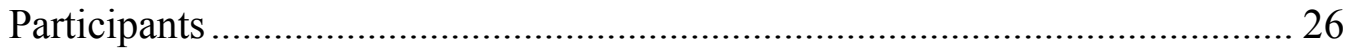

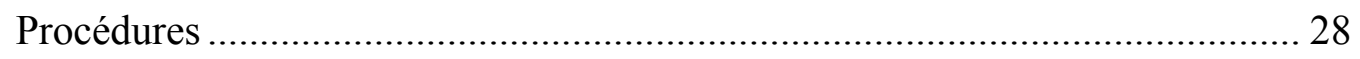

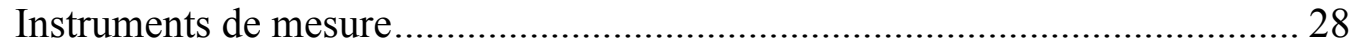

Mémoire de travail auditivo-verbale...............................................28

Mémoire de travail visuospatiale ...................................................28 
Inhibition et attention

Raisonnement non verbal..........................................................29

Compréhension de lecture et raisonnement mathématique ....................30

Fonctions exécutives au quotidien ............................................... 30

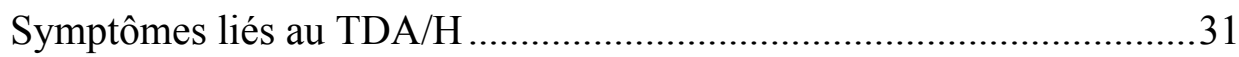

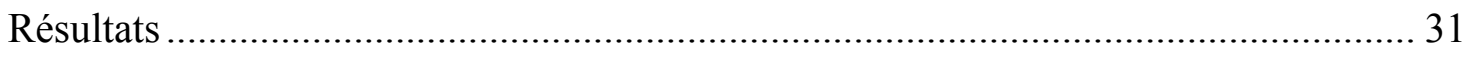

Mémoire de travail ............................................................................... 34

Raisonnement non verbal, fonctions attentionnelles et inhibition .................. 35

Fonctionnement exécutif quotidien et symptômes associés au TDA/H............ 36

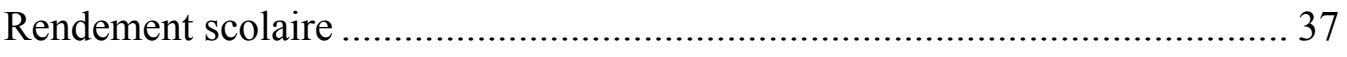

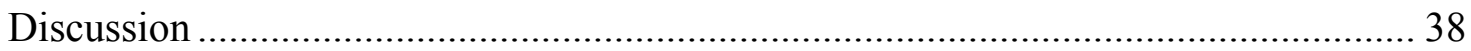

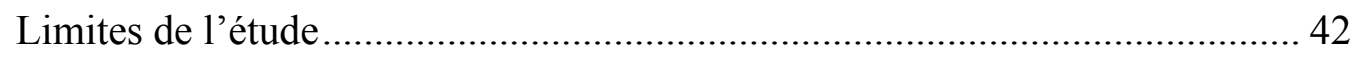

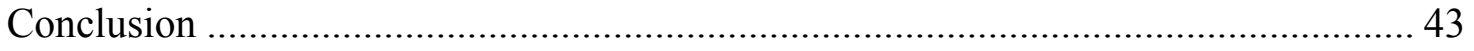

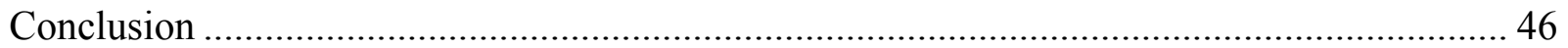

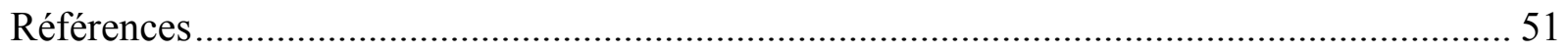




\section{Liste des tableaux}

Tableau 1 Programme Cogmed et TDA/H : description des études avec suivi à long terme.... 21

Tableau 2 Statistiques descriptives des participants ..................................................... 27

Tableau 3 Résultats de l'ANOVA mixte - effets d'interaction et effets principaux ................ 33 


\section{Liste des figures}

Figure 1. Effet principal du temps pour la mémoire de travail auditivo-verbale ................... 34

Figure 2. Effet principal du temps pour la mémoire de travail visuospatiale ....................... 35

Figure 3. Effet principal du temps pour le raisonnement non verbal ................................. 36

Figure 4. Effet principal du temps pour le raisonnement mathématique ............................. 37 


\section{Liste des abréviations}

ADHD: Attention Deficit Hyperactivity Disorder

BRIEF: Behavior Rating of Executive Function

CPM: Colored Progressive Matrices

CPT-II: Continuous Performance Test $-2^{\text {nd }}$ edition

CWMT : Cogmed Working Memory Training

DSM-5 : Manuel diagnostique et statistique des troubles mentaux de l'Association Américaine de Psychiatrie, cinquième édition

MdT : Mémoire de travail

TDA/H : Trouble déficitaire de l'attention avec ou sans hyperactivité

WIAT-II: Wechsler Individual Achievement Test $-2^{\text {nd }}$ edition

WISC-IV: Wechsler Intelligence Scale for Children $-4^{\text {th }}$ edition

WM: Working Memory

WNV: Wechsler Nonverbal Scale of Ability 


\section{Remerciements}

Je tiens d'abord à remercier mon directeur d'essai doctoral, Bruno Gauthier. Merci pour ton soutien continu, ta bienveillance et ta confiance. J'aimerais également remercier tous ceux ayant été impliqués de près ou de loin dans ce projet. Merci pour votre aide et vos précieux conseils. Un merci particulier à Amélie Dentz et aux membres de l'équipe professorale pour m'avoir guidée à travers ce projet. Un grand merci à mes collègues qui m’ont encouragé tout au long de mon doctorat. Merci pour votre grande disponibilité et votre écoute inconditionnelle. Finalement, je tiens à remercier tous ceux et celles qui m'ont épaulé et qui m'ont encouragé à persévérer lorsque nécessaire. 


\section{Introduction}

Le TDA/H est un trouble neurodéveloppemental caractérisé par des comportements d'inattention, d'hyperactivité et d'impulsivité ne correspondant pas au stade développemental de l'enfant (DSM-5 ; American Psychiatric Association [APA], 2013). Le DSM-5 propose trois présentations possibles du TDA/H: à prédominance inattentive, à prédominance hyperactiveimpulsive ou mixte. Un diagnostic est émis lorsque les comportements d'inattention, d'hyperactivité et d'impulsivité débutent avant l'âge de 12 ans, persistent au moins six mois et entrainent un dysfonctionnement dans au moins deux des domaines suivants : social, familial ou scolaire (APA, 2013). La prévalence mondiale du TDA/H est estimée à 5\% des enfants d'âge scolaire, ce qui en fait un des troubles neurodéveloppementaux les plus fréquents (Polanczyk, Willcutt, Salum, Hieling et Rohde, 2014). Ce trouble persiste à l'âge adulte dans plus de 50\% des cas (Biederman et al., 2006; 2012) et s'accompagne d'un risque élevé de comorbidité(s): trouble d'apprentissage, trouble d'opposition avec provocation, troubles des conduites, troubles anxieux, troubles de langage, etc. (Elia, Ambrosini et Berrettini, 2008 ; Farias et al., 2017; Larson, Russ, Kahn et Halfon, 2011). Le TDA/H est aussi le trouble comorbide le plus commun dans le contexte d'un syndrome de Gilles de la Tourette (Rizzo, Gulisano, Calì et Curatolo, 2013).

Le TDA/H est une condition chronique et invalidante qui interfère de façon significative avec le fonctionnement académique/professionnel, social et/ou familial (APA, 2013). Il a un impact majeur sur les activités de la vie quotidienne, notamment sur le plan des relations interpersonnelles, de l'éducation, du fonctionnement familial et occupationnel, de l'autonomie et du respect des normes sociales, des règles et des lois (Barkley, 2002). À long terme, ce trouble est associé à un risque important d'échecs scolaires, de problèmes sociaux, de troubles de santé mentale (anxiété, dépression, dépendance) et de délinquance (Biederman et al., 2006). De par ses 
répercussions négatives sur le fonctionnement cognitif, émotionnel, social, familial et académique/professionnel, le TDA/H affecte considérablement la qualité de vie, le bien-être et l'estime de soi des individus qui en sont atteints (Wehmeier et al., 2010). De plus, il engendre des coûts importants pour les familles et la société, le classant ainsi parmi les problèmes de santé publique majeurs (Polanczyk et al., 2014).

Compte tenu des conséquences sévères associées à ce trouble, il s'avère fondamental de mettre en place des stratégies d'intervention efficaces dès l'enfance (Biederman et al., 2012). À cet effet, de plus en plus d'efforts sont investis afin de cibler spécifiquement les déficits neuropsychologiques sous-jacents au TDA/H, et ce, dans le but d'obtenir des bénéfices plus larges et persistants (Qian et al., 2017). L'entrainement cognitif est une intervention proposée en ce sens (Rapport et al., 2013) et sera abordé plus en profondeur dans les prochaines sections.

\section{Neuropsychologie du TDA/H}

Le TDA/H est un trouble complexe et hétérogène se caractérisant par de multiples anomalies sur les plans neuro-anatomique, neurophysiologique et neuro-fonctionnel (Nigg, Willcutt, Doyle et Sonuga-Barke, 2005). À ce jour, le TDA/H est perçu comme un trouble reflétant des atteintes de connectivité entre les réseaux neuronaux impliqués dans l'attention, le contrôle exécutif, la régulation émotionnelle et la mémoire de travail (Arnsten et Rubia, 2012; Cortese et al., 2012). Les corrélats neuronaux du TDA/H mis en évidence par une méta-analyse révèlent plus particulièrement un dysfonctionnement des réseaux cérébello-striato-préfrontaux (Cortese et al., 2012) impliquant des régions cérébrales telles que le cortex préfrontal, le cortex cingulaire antérieur, les ganglions de la base, le thalamus, le cervelet et le splenium du corps calleux (Dickstein, Bannon, Castellanos et Milham, 2006; Friedman et al., 2015; Valera, Faraone, Murray et Seidman, 2007). Les résultats d'une étude en neuro-imagerie montrent entre 
autres que les enfants atteints d'un TDA/H accusent un retard de maturation des aires frontales, tel que mesuré par l'épaisseur et l'aire corticale (Shaw et al., 2007 ; 2012). Au niveau neurofonctionnel, le TDA/H serait caractérisé par une sous-activation des circuits cérébraux impliqués dans les fonctions exécutives et l'attention et une hyper-activation du réseau par défaut, du système moteur et du système visuel (Cortese et al., 2012). Cette hyper-activation témoignerait vraisemblablement de mécanismes compensatoires sous-jacents (Cortese et al., 2012).

Plusieurs modèles neurocognitifs du TDA/H ont été avancés à travers les années. L'hétérogénéité des profils neuropsychologiques caractérisant le TDA/H (Karalunas et al., 2017) a cependant donné lieu à un changement de paradigme dans la modélisation de ce trouble, avec notamment l'apparition des modèles à plusieurs voies (Sonuga-Barke, 2005). Le modèle unitaire de Barkley (1997), selon lequel le TDA/H découle d'un déficit primaire du contrôle inhibiteur, est quant à lui remis en question depuis plusieurs années. En effet, selon une méta-analyse importante, les atteintes exécutives au cœur du modèle de Barkley ne seraient ni nécessaires, ni suffisantes pour expliquer ce trouble (Willcutt, Doyle, Nigg, Faraone et Pennington, 2005). De fait, 30 à $50 \%$ des enfants diagnostiqués avec un TDA/H ne présenteraient pas de déficits objectivables à l'endroit des fonctions exécutives (Van Hulst, Zeeuw et Durston, 2015). Plus récemment, Sonuga-Barke, Bitsakou et Thompson (2010) ont proposé un modèle à trois voies reflétant trois sous-types plus ou moins distincts de TDA/H. La première voie renvoie à un défaut d'inhibition impliquant le réseau méso-cortical de la dopamine et ses projections vers le cortex préfrontal. La deuxième voie renvoie plutôt à un style motivationnel caractérisé par une aversion du délai résultant d'une altération du circuit de la récompense. La troisième voie renvoie quant à elle à des déficits au niveau du timing, c'est-à-dire au niveau de la gestion et de l'estimation du temps. Un modèle similaire fut avancé par Durston et ses collaborateurs (2011). 
Ces auteurs proposent un modèle à voies multiples où trois profils cognitifs relativement indépendants sont définis en fonction des circuits neurobiologiques impliqués : le circuit « dorsal fronto-striatial » lié au contrôle cognitif, le circuit « orbitofronto-striatal » lié au circuit de la récompense et/ou le circuit « fronto-cérébelleux » lié au timing. Ces modèles à voies multiples permettraient donc de distinguer un sous-type exécutif, un sous-type motivationnel et un soustype temporel selon les domaines cognitifs principalement touchés.

Sur le plan cognitif, la mémoire de travail (MdT) serait l'une des principales fonctions affectées dans le contexte d'un TDA/H (Kasper, Alderson et Hudec, 2012). De nombreux modèles de la MdT existent à l'heure actuelle, le plus utilisé dans le cadre de la recherche sur le TDA/H étant le modèle à trois composantes de Baddeley (Kasper et al., 2012). La MdT telle que conçue par le modèle de Baddeley (1974) réfère à un système à capacité limitée responsable du stockage temporaire, de la rétention et de la manipulation mentale de l'information. Cette fonction joue un rôle crucial dans la régulation comportementale et influence plusieurs habiletés intellectuelles et académiques, dont la lecture et les mathématiques (Baddeley, 2012; Swanson et Kim, 2007). L'administrateur central réfère à la composante exécutive de la MdT et serait la composante la plus atteinte dans le cadre d'un TDA/H (Kasper et al., 2012). Celui-ci est défini comme un système de contrôle permettant de coordonner des tâches, d'inhiber les distracteurs et de manipuler les informations activées en mémoire à court terme. Il contrôle la boucle phonologique et le calepin visuospatial, soit les deux sous-composantes associées au modèle qui sont impliquées dans le stockage à court terme des informations verbales et non-verbales, respectivement.

Les déficits observés au niveau de la MdT, particulièrement au niveau de l'administrateur central, seraient corrélés avec les symptômes d'inattention (Burgess et al., 2010), d'hyperactivité 
(Rapport et al., 2009), d'impulsivité (Raiker, Rapport, Kofler et Sarver, 2012) et les problèmes sociaux (Kofler et al., 2011). Ils seraient à l'origine des difficultés de concentration et d'apprentissage souvent vécues dans le contexte d'un TDA/H (Sjöwall et Thorell, 2014; Söderqvist et Nutley, 2015). En outre, un déficit de la MdT peut nuire à certaines habiletés comme le raisonnement, la planification et la résolution de problèmes (Dovis, Oord, Wiers et Prins, 2013), de même qu'engendrer une diminution des performances scolaires (Qian et al., 2017; Rogers, Hwang, Toplak, Weiss et Tannock, 2011). Selon Barkley (1997), les problèmes de MdT sont secondaires au défaut d'inhibition central au TDA/H et s'inscrivent dans un contexte plus général d'atteintes sur le plan exécutif. Le modèle à plusieurs voies de Sonuga-Barke et al. (2010) associe quant à lui les problèmes de MdT liés au TDA/H à un sous-type exécutif impliquant la voie méso-corticale du circuit dopaminergique. De leur côté, Durston et ses collaborateurs (2011) réfèrent au circuit dorsal fronto-striatal lié au contrôle cognitif pour expliquer les difficultés de MdT.

\section{Traitement du TDA/H}

À ce jour, la pharmacothérapie est considérée comme étant le traitement le plus efficace du TDA/H (American Academy of Pediatrics [AAP], 2011; Faraone et Buitelaar, 2010). Bien que la médication soit indiquée comme traitement de première ligne, une approche multimodale intégrant des interventions psychosociales est généralement recommandée (AAP, 2011). Malgré leurs impacts favorables dans plusieurs sphères de fonctionnement, ces interventions font face à certaines limites (Cortese et al., 2015). En effet, une minorité importante des jeunes atteints d'un $\mathrm{TDA} / \mathrm{H}$ ne répondent que partiellement à la médication psychostimulante (Faraone, Biederman, Spencer et Aleardi, 2006) ou expérimentent des effets secondaires désagréables compromettant la poursuite du traitement (Cortese et al., 2013; Graham et Coghill, 2008). Par ailleurs, les effets 
bénéfiques de la médication sur les symptômes liés au TDA/H ne persistent pas une fois l'arrêt du traitement (Molina et al., 2009). Les interventions comportementales sont quant à elles plus difficiles à maintenir dans le temps, généralement plus coûteuses et, selon certains, moins efficaces que la médication (Chacko et al., 2013; Sonuga-Barke et al., 2013; Van der Oord, Prins, Oosterlaan et Emmelkamp, 2008). De plus, selon une méta-analyse, les interventions psychosociales et pharmacologiques ne procurent pas d'amélioration significative des performances scolaires, alors qu'il s'agit d'un enjeux majeur associé au TDA/H (Van der Oord et al., 2008). Les limites soulevées ci-dessus mettent en lumière le besoin important de développer de nouvelles interventions alternatives ou complémentaires à la prise en charge psychopharmacologique du TDA/H (Qian et al., 2017). La remédiation cognitive sous forme d'entrainement cognitif assisté par ordinateur est une intervention proposée dans cette optique et suscite un grand intérêt auprès des chercheurs (Fuermaier et al., 2017; Rosa et al., 2017).

\section{L'entrainement cognitif}

L'entrainement cognitif s'inscrit dans une perspective de prise en charge des troubles neuropsychologiques sous-jacents au TDA/H et vise le développement de fonctions cognitives déficitaires par la répétition d'exercices adaptés aux performances de l'individu et réalisés durant plusieurs semaines (Rutledge, van den Bos, McClure et Schweitzer, 2012). Cette approche s'appuie sur le concept de plasticité cérébrale et a été développée selon l'approche « bottom-up » (Klingberg, 2010 ; Vinogradov, Fisher et de Villers-Sidani, 2012). Les techniques impliquées par l'entrainement cognitif se fondent sur le modèle de Hebb et reposent sur la prémisse selon laquelle l'activation répétée d'une fonction augmente sa capacité et facilite la réorganisation corticale des réseaux neuronaux qui sous-tendent les processus cognitifs ciblés (Deforge, 2011). En ciblant les déficits cognitifs généralement associés au TDA/H, l'entrainement cognitif 
permettrait de renforcer les circuits neuronaux sous-jacents et, par le fait même, de réduire les symptômes associés au TDA/H et d'améliorer le fonctionnement quotidien (Klingberg, 2010). Les recherches montrent en effet plusieurs bénéfices associés à ce genre d'intervention, notamment à l'égard de la symptomatologie du TDA/H (Rutledge et al., 2012).

En tant que déficit majeur associé au TDA/H, la MdT est une fonction souvent ciblée par l'entrainement cognitif (Rapport et al., 2013). Autrefois considérée comme un trait fixe, la MdT serait plutôt malléable grâce à la plasticité des aires cérébrales qui la sous-tendent (Klingberg, Forssberg et Westerberg, 2002; Olesen, Westerberg et Klingberg, 2004; Klingberg et al., 2005; Westerberg et Klingberg, 2007). Selon ces auteurs, le fait d'entrainer la MdT résulterait en une augmentation de sa capacité, laquelle serait un prérequis à l'amélioration des symptômes du TDA/H. La neuro-plasticité de cette fonction permettrait en retour une généralisation ou un transfert des effets de l'entrainement cognitif à d'autres fonctions cognitives liées à la MdT comme l'attention, la compréhension de lecture, les habiletés mathématiques et le raisonnement non verbal (Klingberg, 2010; Rosa et al., 2017).

Le programme Cogmed. Parmi les interventions développées au cours des dernières années, le programme informatisé Cogmed s'avère actuellement le plus utilisé et étudié (Rapport et al., 2013). Ce programme offre un entrainement axé directement sur la MdT et vise l'amélioration de cette fonction via la répétition d'exercices à l'ordinateur (Klingberg, 2010). Il a été développé selon le modèle de la MdT à trois composantes de Baddeley (1974) et comprend 12 exercices sollicitant le calepin visuospatial, la boucle phonologique et/ou l'administrateur central. Ces exercices sont répétés pendant 30-45 minutes, cinq fois par semaine, durant cinq à six semaines consécutives, pour un total d'environ 25 séances. Le programme est conçu pour offrir une rétroaction verbale immédiate au participant afin de lui signaler ses réussites et ses 
erreurs et d'encourager ses efforts. Une description des résultats est également visible à l'écran, permettant au participant de suivre son avancement au fur et à mesure. Le niveau de difficulté, qui correspond au nombre d'éléments à rappeler, est ajusté en fonction de la performance de l'enfant et donc adapté à la capacité de sa MdT. Il existe également une version de comparaison (ou placebo) du programme Cogmed. Cette version offre les mêmes exercices que la version officielle et se déroule dans les mêmes conditions, mais diffère au niveau de l'intensité. Le niveau de difficulté demeure faible et constant (2-3 items) à travers le temps afin de réduire l'efficacité de l'intervention.

Les séances d'entrainement se déroulent habituellement au domicile et sont supervisées par une personne soutien, parent ou autre adulte, afin d'assurer la qualité de l'entrainement et soutenir la motivation du jeune. La personne soutien s'engage à procurer des renforcements positifs à l'enfant, notamment sous forme d'encouragements, ainsi qu'à instaurer un système de récompense. Les progrès de l'enfant sont suivis de près par un entraineur qualifié qui vérifie les résultats et s'assure de l'adhérence du participant via la plateforme Internet Cogmed. Les différents indices disponibles (p. ex., temps d'entrainement par séance, nombre de réussites, etc.) permettent d'offrir un suivi individualisé et adapté au jeune. L'entraineur contacte le participant sur une base hebdomadaire afin de lui faire part de l'évolution de ses performances, répondre à ses questions et l'encourager à poursuivre l'entrainement. Le rôle de la personne soutien et de l'entraineur Cogmed est jugé essentiel dans la mesure où les enfants présentant un TDA/H ont généralement plus de problèmes motivationnels pouvant entraver le bon déroulement de l'entrainement (Chacko et al., 2013).

Efficacité du programme Cogmed. Plusieurs méta-analyses et revues de la littérature démontrent une amélioration de la MdT grâce au programme Cogmed (Chacko et al., 2013; 
Cortese et al., 2015; Dentz, Parent, Gauthier, Guay et Romo, 2016; Schwaighofer, Fischer et Bühner, 2015 ; McLaughlin et al., 2016 ; Shinaver, Entwistle et Söderqvist, 2014 ; SpencerSmith et Klingberg, 2015). Cette intervention s'est révélée efficace auprès d'enfants, d'adolescents et d'adultes présentant diverses pathologies : TDA/H, traumatisme crânien, enfants nés prématurément et enfants avec limite intellectuelle (Söderqvist et Nutley, 2015). Les gains au niveau des capacités de MdT suivant l'entrainement Cogmed s'accompagneraient de changements sur le plan neurophysiologique (Stevens, Gaynor, Bessette et Pearlson, 2016; Thompson, Waskom et Gabrieli, 2016). Certains auteurs observent une augmentation de l'activité cérébrale dans les cortex préfrontal et pariétal et dans les ganglions de la base (Olesen et al., 2004 ; Westerberg et Klingberg, 2007), des changements au niveau de la densité des récepteurs dopaminergiques D1 (McNab et al., 2009) ainsi qu'au niveau de la connectivité au repos de certaines aires cérébrales impliquées dans la MdT (Astle, Barnes, Baker, Colclough et Woolrich, 2015). Notons cependant que ces études comportent uniquement des échantillons neurotypiques et principalement adultes. Ces effets neurophysiologiques ne sont donc pas attestés auprès des jeunes présentant un TDA/H.

Klingberg et ses collègues (2002 et 2005) sont les premiers auteurs à avoir démontré l'efficacité du programme Cogmed auprès d'enfants présentant un TDA/H. Leurs résultats révélaient une amélioration significative de la MdT ainsi qu'un transfert des effets du programme sur l'inhibition et le raisonnement non verbal. Plusieurs études ont tenté de répliquer ces résultats auprès d'une population TDA/H (Beck et al., 2010; Chacko et al., 2014; DongenBoosma, Vollebregt, Buitelaar et Slaats-Willemse, 2014; Egeland, Aarlien et Saunes, 2013; Gibson et al., 2011; Gray et al., 2012; Green et al., 2012; Gropper, Gotlieb, Kronitz et Tannock, 2014; Holmes et al., 2010; Hovik, Saunes, Aarlien et Egeland, 2013; Mezzacappa et Buckner, 
2010; Stevens, Gaynor, Bessette et Pearlson, 2015; Van der Donk, Hiemstra-Beernink, TjeenkKalff, Van der Leij et Lindauer, 2015). Bien que plusieurs études confirment les bienfaits immédiats de cette intervention sur la MdT, certaines méta-analyses mettent en lumière une divergence importante des résultats quant aux effets de transfert du programme Cogmed et remettent en question son efficacité par rapport au traitement du TDA/H (Chacko et al., 2013; Melby-Lervåg et Hulme, 2013; Shipstead, Hick et Engel, 2012; Rapport et al., 2013). L'efficacité à plus long terme de cette intervention soulève d'autant plus de préoccupations qu'elle n'est pas systématiquement étudiée, ni clairement démontrée.

Selon une recension des écrits réalisée par Dentz et ses collaborateurs (2016), les effets du programme Cogmed sur la MdT auditivo-verbale et visuospatiale sont faibles à modérés auprès d'enfants et d'adolescents présentant un TDA/H. Le transfert des effets sur des fonctions cognitives non ciblées par l'entrainement telles que l'attention, l'inhibition et le raisonnement non verbal n'est quant à lui pas démontré. La généralisation des effets du programme Cogmed sur les symptômes liés au TDA/H et sur le rendement scolaire est également controversée (Dentz et al., 2016). Certaines études suggèrent un impact favorable du programme Cogmed sur les problèmes d'attention et d'hyperactivité/impulsivité rapportés à l'aide de questionnaires remplis par les parents (Klingberg et al., 2005 ; Beck et al., 2010), alors qu'à l'inverse d'autres études ne trouvent pas d'effets (Chacko et al., 2014 ; Egeland et al., 2013 ; Gray et al., 2012 ; Green et al., 2012 ; Van Dongen-Boosma et al., 2014). Dans l'ensemble, aucune amélioration significative des performances scolaires en lecture et en mathématique n'est rapportée à la suite de l'entrainement (Chacko et al., 2014 ; Gray et al., 2012 ; Gropper et al., 2014). Le maintien des acquis à plus long terme n'est pas attesté (Dentz et al., 2016). 
En 2016, McLaughlin et collaborateurs réalisaient une méta-analyse sur l'efficacité du programme Cogmed incluant 13 études publiées entre 2011 et 2015. Parmi celles-ci, 4 études ciblaient les effets du programme Cogmed chez les enfants et les adolescents présentant un TDA/H (Chacko et al., 2014; Green et al., 2012; Hovik et al., 2013; Van Dongen-Boosma et al., 2014). Les résultats de cette méta-analyse suggèrent que le programme Cogmed a un léger impact sur la MdT auditivo-verbale et visuospatiale, et ce, tel que mesuré par des tâches s'apparentant à celles employées durant les séances d'entrainement. Aucune amélioration du rendement académique (c.-à-d., habiletés de lecture et de mathématique) n'est objectivée. Les résultats indiquent cependant une diminution significative des symptômes d'inattention et d'hyperactivité. L'absence d'un groupe contrôle actif et d'un design en double aveugle au sein de plusieurs études limite cependant l'interprétation des résultats. En effet, certains résultats pourraient être attribuables à un effet placebo dans la mesure où les attentes des participants et de leurs parents risquent d'être biaisées en faveur de l'intervention de par leur implication même. En ce qui a trait au maintien des acquis, seulement 4 études sur 13 comprenaient des mesures à plus long terme (Brehmer, Westerberg et Bäckman, 2012; Hovik et al., 2013; Mawjee, Woltering et Tannock, 2015; Söderqvist et Nutley, 2015). Bien qu'ils déplorent le manque d'études récentes s'intéressant aux effets à plus long terme du programme Cogmed, les auteurs n'ont pas examiné le maintien des acquis dans le cadre de la présente méta-analyse.

Plusieurs limites sur le plan méthodologique, dont un faible effectif, l'absence d'un groupe contrôle actif et d'évaluations réalisées en double insu, ont été soulevées dans diverses méta-analyses et peuvent expliquer la divergence des résultats (Chacko et al., 2013; MelbyLervåg et Hulme, 2013; Shipstead, Hick et Engel, 2012; Rapport et al., 2013). L'hétérogénéité des participants d'une étude à l'autre limite également la portée des résultats. En effet, peu 
d'études tiennent compte des caractéristiques des participants (p. ex., âge, sexe, troubles associés) et les critères d'inclusion et d'exclusion varient considérablement entre les études (Van der Donk, Hiemstra-Beernink, Tjeenk-Kalff, Van der Leij et Lindauer, 2016). De plus, très peu d'études s'intéressent à l'apport supplémentaire de l'entrainement cognitif par rapport à la pharmacothérapie (Rosa et al., 2017). À notre connaissance, une seule étude compare les effets complémentaires du programme Cogmed à ceux de la médication (Holmes et al., 2010). Les résultats indiquent que la réalisation du programme Cogmed conjointement à la prise de médication permettraient d'améliorer les deux composantes de la MdT, alors que la médication employée seule permettrait seulement d'améliorer la composante visuospatiale.

Compte tenu de la popularité croissante du programme Cogmed et de l'usage commercial qui en est fait, il s'avère essentiel d'en attester l'efficacité à plus long terme. En général, les effets du programme Cogmed sur la MdT tendent à persister 2 à 12 mois après l'intervention (Söderqvist et Nutley, 2015). À l'heure actuelle, six études ont investigué le maintien des effets du programme Cogmed auprès d'enfants et d'adolescents présentant un TDA/H (Beck, Hanson, Puffenberger, Benninger et Benninger, 2010; Bigorra, Garolera, Guijarro et Hervás, 2016; Holmes et al., 2010; Hovik et al., 2013 ; Klingberg et al., 2005; Van der Donk et al., 2015). Les résultats indiquent dans l'ensemble qu'il y a maintien des acquis 3 à 8 mois post-intervention sur la MdT. En revanche, aucun consensus ne semble émerger de la littérature quant au transfert et à la généralisation des effets du programme au long cours sur le reste du fonctionnement cognitif, les symptômes liés au TDA/H et les performances scolaires. De plus, plusieurs limites à l'interprétation des résultats ont été soulevées à travers différentes méta-analyses (Chacko et al., 2013; Dentz et al., 2016; McLauglin et al., 2016; Melby-Lervåg et Hulme, 2013; Shipstead et al., 2012; Rapport et al., 2013). Ces auteurs déplorent le manque d'études incluant un groupe 
contrôle actif et des évaluations réalisées en double insu et tenant compte des caractéristiques des participants, notamment en ce qui a trait à la présentation du TDA/H, la prise de médication et la présence de troubles comorbides. De telles études sont jugées nécessaires afin de réellement démontrer l'efficacité du programme Cogmed dans le traitement du TDA/H.

L'article empirique en préparation pour publication sera présenté dans les prochaines pages. Cet article porte sur les effets à plus long terme du programme Cogmed et cherche à examiner l'efficacité au long cours de cette intervention auprès de jeunes présentant un TDA/H mixte avec comorbidités. Il vise également à explorer l'apport complémentaire de l'entrainement cognitif lorsqu'il est réalisé en concomitance avec un traitement pharmacologique. L'article débutera par une introduction permettant de mieux comprendre la problématique et sera ensuite suivi des objectifs de l'étude. La méthodologie, les résultats puis la discussion seront finalement exposés. Une conclusion générale revenant sur les principaux enjeux soulevés par la présente étude et sur certaines pistes de réflexion suivra finalement l'article. 


\title{
Suivi à long terme des effets d'un entrainement de la mémoire de travail (Cogmed) auprès d'enfants présentant un TDA/H
}

\author{
Camille Rivard, B.Sc., Amélie Dentz, Ph.D., Lucia Romo, Ph.D., Véronique Parent, \\ Ph.D., Marie-Claude Guay, Ph.D., Bruno Gauthier, Ph.D.
}

Camille Rivard, B.Sc., Département de psychologie, Université de Montréal, Montréal, Québec, Canada

Amélie Dentz, Ph.D., Université Paris Nanterre, France

Lucia Romo, Ph. D., Professeure, Université Paris Nanterre, France

Véronique Parent, Ph. D., Professeure, Université de Sherbrooke, Canada

Marie-Claude Guay, Ph. D., Professeure, Université du Québec à Montréal, Canada

Bruno Gauthier, Ph.D., Département de psychologie, Université de Montréal, Montréal, Québec, Canada

Manuscrit en préparation pour soumission dans Revue canadienne de psychologie expérimentale 


\section{Résumé}

Introduction : L'entrainement de la mémoire de travail par le programme Cogmed est une intervention proposée dans le cadre de la prise en charge des jeunes présentant un trouble du déficit de l'attention/hyperactivité (TDA/H). L'efficacité à plus long terme de ce type d'intervention n'est cependant pas clairement démontrée à l'heure actuelle. Objectif : Examiner l'impact à long terme du programme Cogmed sur la mémoire de travail, l'attention, l'inhibition, le raisonnement non verbal, le rendement scolaire, le fonctionnement exécutif quotidien et les symptômes associés au TDA/H. Méthode : La présente étude comprend 31 participants âgés de 7 à 13 ans présentant un TDA/H mixte associé à diverses comorbidités et recevant un traitement pharmacologique stable. Les participants ayant d'abord complété le programme Cogmed dans le cadre de l'étude de Dentz et ses collaborateurs (soumis) ont pris part à une évaluation cognitive six mois post-intervention. Résultats : Le programme Cogmed n'améliore pas davantage les capacités de mémoire de travail du groupe expérimental, comparativement au groupe contrôle actif, lorsqu'ils sont évalués six mois post-intervention. Aucun effet de transfert n'émerge avec le passage du temps. Conclusion : Les résultats ne soutiennent pas l'efficacité du programme Cogmed à long terme et ne permettent pas de recommander son utilisation de façon complémentaire à la médication auprès d'enfants atteints d'un TDA/H mixte avec comorbidité(s). Augmenter la valeur écologique des programmes d'entrainement cognitif pourrait favoriser les apprentissages, leur généralisation en contexte réel et le maintien des acquis dans le temps. D'autres études sont à envisager afin d'identifier les conditions permettant d'atteindre un niveau d'efficacité optimale, à court comme à plus long terme.

Mots-clés : Entraînement cognitif, Mémoire de travail, Programme Cogmed, Maintien des acquis, Trouble du déficit de l'attention/hyperactivité (TDAH), Neuropsychologie clinique 


\section{Introduction}

Le trouble du déficit de l'attention avec ou sans hyperactivité (TDA/H) est une condition neurodéveloppementale caractérisée par des symptômes persistants d'inattention et/ou d’hyperactivité-impulsivité (DSM-5 ; American Psychiatric Association [APA], 2013). La prévalence mondiale du TDA/H oscille entre 5\% et 7\% des enfants d'âge scolaire (Polanczyk, Willcutt, Salum, Hieling et Rohde, 2014). Il s'agit d'un trouble chronique associé à un risque élevé de comorbidités dont les principales sont : les troubles d'apprentissage (Farias et al., 2017), des conduites, de l'humeur (anxiété, dépression) ou du langage (Larson, Russ, Kahn et Halfon, 2011), le trouble d'opposition avec provocation (Elia, Ambrosini et Berrettini, 2008) et le syndrome de Gilles de la Tourette (Rizzo, Gulisano, Calì et Curatolo, 2013). Les divers déficits mis en évidence sur le plan cognitif, notamment en lien avec l'autorégulation comportementale/ émotionnelle (Sjöwall, Roth, Lindqvist et Thorell, 2013) et la mémoire de travail (MdT) (Karalunas et al., 2017), altèrent le fonctionnement quotidien et affectent le bien-être et l'estime de soi des individus qui en sont atteints (Sjöwall et Thorell, 2014; Wehmeier, Schacht et Barkley, 2010). À long terme, ce trouble est associé à un risque important d'échecs scolaires, de problèmes sociaux, de troubles de santé mentale et de délinquance (Biederman et al., 2006, 2012). De par ses diverses répercussions sur les sphères sociale, académique et/ou familiale, le TDA/H entraine des coûts importants pour la famille et la société et se classe ainsi parmi les

problèmes majeurs de santé publique (Polanczyk et al., 2014). Dans cette perspective, il s'avère primordial de mettre en place des stratégies de prévention et d'intervention efficaces dès l'enfance.

Le traitement du TDA/H, de par sa nature complexe et hétérogène (Nigg, Willcutt, Doyle et Sonuga-Barke, 2005), implique habituellement une approche multimodale intégrant des axes 
thérapeutiques variés (Seixas, Weiss et Müller, 2012). Certains auteurs recommandent en ce sens de combiner la médication à un programme d'entrainement cognitif, tel l'entrainement de la mémoire de travail, dans le but d'accroître les effets de la médication sur le plan cognitif (Fuermaier et al., 2017). En effet, si certaines études montrent des effets positifs associés à la médication sur le fonctionnement attentionnel et exécutif (Bolfer et al., 2017), d'autres études obtiennent des résultats plus limités à cet égard (Advokat, 2010). La prise de médication durant l'entrainement cognitif permettrait de couvrir un plus vaste éventail de symptômes et de bonifier les effets du programme d'entrainement cognitif (Rosa et al., 2017). La concomitance de ces deux types d'intervention permettrait entre autres d'améliorer davantage la MdT qu'un traitement pharmacologique à lui seul (Holmes et al., 2010).

L'entrainement cognitif s'appuie sur le concept de plasticité cérébrale (Vinogradov, Fisher et de Villers-Sidani, 2012) et vise la restauration de fonctions cognitives par la pratique répétée et intensive d'exercices généralement informatisés de complexité croissante (Rutledge, van den Bos, McClure et Schweitzer, 2012). Cette approche repose sur la prémisse qu'en ciblant les déficits neuropsychologiques associés au TDA/H, l'entrainement cognitif permettrait de renforcer les circuits neuronaux sous-jacents et, par le fait même, de réduire les symptômes associés au TDA/H et d'améliorer le fonctionnement quotidien (Cortese et al., 2015; Qian et al., 2017). La neuro-plasticité permettrait en effet un transfert des acquis vers d'autres domaines n'étant pas directement ciblés par le programme comme le fonctionnement comportemental quotidien et le rendement scolaire (Klingberg, 2010). Pour parvenir à cette réorganisation structuro-fonctionnelle, les principes d'adaptabilité et d'intensivité sont considérés comme étant essentiels (Klingberg, Forssberg et Westerberg, 2002). En effet, l'ajustement du niveau de difficulté en fonction des capacités de l'individu et le caractère intensif de la prise en charge 
permettraient d'optimiser les effets de l'entrainement (Deforge, 2011).

En tant que déficit majeur associé au TDA/H (Kasper, Alderson et Hudec, 2012), la MdT est une fonction souvent ciblée par l'entrainement cognitif (Rapport et al., 2013). La neuroplasticité de cette fonction permettrait d'influencer d'autres processus cognitifs et serait particulièrement propice à la généralisation des effets (Rosa et al., 2017). Selon le modèle de Baddeley (1974), la MdT réfère à un système à capacité limitée responsable du stockage temporaire, de la rétention et de la manipulation mentale de l'information. L'administrateur central est défini comme un système de contrôle permettant de coordonner des tâches, d'inhiber les distracteurs, de manipuler les informations activées en mémoire à court terme. Il contrôle la boucle phonologique et le calepin visuospatial, soit deux sous-composantes associées au modèle qui sont impliquées dans le stockage temporaire des informations verbales et non-verbales, respectivement. Ces sous-systèmes soutiendraient deux types distincts de MdT, soit la MdT auditivo-verbale et la MdT visuospatiale.

La MdT est étroitement liée aux capacités attentionnelles et joue un rôle critique dans la régulation comportementale (Alloway et Copello, 2013; Martinussen, Hayden, Hogg-Johnson et Tannock, 2005). Une faible capacité de MdT peut donc entrainer des difficultés à suivre des instructions (Yang, Allen et Gathercole, 2016) et à garder en tête le but d'une tâche à accomplir (Dovis, Van der Oord, Wiers et Prins, 2013). Cette fonction cognitive influence également plusieurs habiletés intellectuelles et académiques, dont la lecture et les mathématiques (Baddeley, 2012; Miller, Nevado-Montenegro et Hinshaw, 2012; Swanson et Kim, 2007). Les problèmes de MdT peuvent nuire à certaines habiletés comme le raisonnement, la planification, la résolution de problèmes (Dovis et al., 2013) et sont associés à un risque accru d'échecs scolaires (Rogers, Hwang, Toplak, Weiss et Tannock, 2011). 
Le programme Cogmed offre un entrainement axé directement sur la MdT et comprend une version spécialement conçue pour les enfants d'âge scolaire (http://www.cogmed.com). Ce logiciel a été développé selon le modèle de la MdT à trois composantes de Baddeley (1974) et comprend 12 exercices sollicitant le calepin visuospatial, la boucle phonologique et/ou l'administrateur central. Ces exercices sont répétés pendant 30-45 minutes, cinq fois par semaine, durant cinq à six semaines consécutives, pour un total d'environ 25 séances. Le programme est conçu pour offrir une rétroaction verbale immédiate au participant afin de lui signaler ses réussites et ses erreurs et d'encourager ses efforts. Une description des résultats est également visible à l'écran, permettant au participant de suivre son avancement. Le niveau de difficulté, qui correspond au nombre d'éléments à rappeler, est ajusté en fonction de la performance de l'enfant et donc adapté à la capacité de sa MdT. Ainsi, lorsqu'un nombre déterminé d'essais est réussi, les essais subséquents comprennent un élément supplémentaire à retenir, ce qui taxe davantage la MdT. À l'inverse, les erreurs s'accompagnent d'une diminution du niveau de difficulté, ce qui implique de retenir un élément de moins et réduit la charge sur la MdT. Il existe également une version placebo du programme Cogmed. Cette version offre les mêmes exercices que la version officielle et se déroule dans les mêmes conditions, mais diffère quant à l'intensité. Le niveau de difficulté demeure faible et constant (2-3 items) à travers le temps afin de réduire l'efficacité de l'intervention. Les séances d'entrainement se déroulent habituellement au domicile et sont supervisées par une personne soutien, parent ou autre adulte, afin d'assurer la qualité de l'entrainement et de soutenir la motivation du jeune. Les progrès du jeune sont suivis de près par un entraineur qualifié. Certains indices en lien avec les paramètres de l'entrainement (p. ex., temps d'entrainement par séance) et la progression du participant (p. ex., nombre de réussites) sont accessibles par le biais de la plateforme Internet Cogmed. 
Plusieurs méta-analyses et revues de la littérature montrent une amélioration de la MdT auditivo-verbale et/ou visuospatiale grâce au programme Cogmed auprès d'enfants et d'adolescents présentant un TDA/H (Chacko et al., 2013; Cortese et al., 2015; Dentz, Parent, Gauthier, Guay et Romo, 2016; Schwaighofer, Fischer et Bühner, 2015 ; McLaughlin et al., 2016 ; Shinaver, Entwistle et Söderqvist, 2014 ; Spencer-Smith et Klingberg, 2015). Néanmoins, de plus en plus d'études mettent en lumière une divergence importante des résultats quant aux effets de transfert associés au programme Cogmed (p. ex., attention, inhibition, rendement académique, symptômes liés au TDA/H) et soulèvent des limites importantes sur le plan méthodologique qui remettent en question l'efficacité de cette intervention dans la prise en charge des jeunes présentant un TDA/H (Chacko et al., 2013; Dentz et al., 2016; McLaughlin et al., 2016; Melby-Lervåg et Hulme, 2013; Shipstead, Redick et Engel, 2012; Rapport et al., 2013). L'évaluation non systématique du maintien des acquis à plus long terme fait partie de ces limites méthodologiques. En effet, les effets attendus du programme peuvent être validés empiriquement à court terme, encore faut-il qu'ils persistent dans le temps pour justifier une telle intervention. De plus, certaines études montrent l'apparition de gains non pas immédiatement après l'entrainement cognitif, mais quelques mois plus tard (Bigorra, Garolera, Guijarro et Hervás, 2016; Holmes, Gathercole et Dunning, 2009; Hovik, Saunes, Aarlien et Egeland, 2013; Van der Molen, Van Luit, Van der Molen, Klugkist et Jongmans, 2010). Il est donc possible que les effets du programme Cogmed ne soient pas immédiats, mais plutôt à retardement (Gathercole, 2014). Une mesure du maintien des acquis sur plusieurs mois s'avère donc essentielle pour réellement démontrer l'efficacité de cette intervention.

À ce jour, six études ont investigué les effets à long terme du programme Cogmed auprès d'enfants présentant un TDA/H (Beck, Hanson, Puffenberger, Benninger et Benninger, 2010; 
Bigorra et al., 2016; Holmes et al., 2010; Hovik et al., 2013 ; Klingberg et al., 2005; van der Donk, Hiemstra-Beernink, Tjeenk-Kalff, Van Der Leij et Lindauer, 2015). Ces études sont décrites sommairement dans le Tableau 1. En revanche, plusieurs études examinant les effets du programme Cogmed chez les jeunes atteints d'un TDA/H ne comprennent pas de mesures à plus long terme (Chacko et al., 2014; Dongen-Boomsma, Vollebregt, Buitelaar et Slaats-Willemse, 2014; Gray et al., 2012; Green et al., 2012; Klingberg, Forssberg et Westerberg, 2002;

Mezzacappa et Buckner, 2010; Stevens, Gaynor, Bessette et Pearlson, 2015).

Tableau 1

Programme Cogmed et TDA/H : description des études avec suivi à long terme

\begin{tabular}{|c|c|c|c|c|c|}
\hline Étude & $\mathbf{N}$ & Âge & Devis & $\begin{array}{c}\text { Suivi } \\
\text { maintien } \\
\text { des acquis }\end{array}$ & Mesures \\
\hline $\begin{array}{l}\text { Klingberg } \\
\text { et al., } \\
2005\end{array}$ & 42 & 7 à 12 & $\begin{array}{l}\text { Assignation aléatoire avec } \\
\text { gp contrôle actif (version } \\
\text { placebo) } \\
\text { Double aveugle }\end{array}$ & 3 mois & $\begin{array}{l}\text { MdT verbale et visuospatiale, } \\
\text { inhibition, raisonnement, } \\
\text { symptômes TDA/H }\end{array}$ \\
\hline $\begin{array}{l}\text { Beck et } \\
\text { al., } 2010\end{array}$ & 52 & 7 à 17 & $\begin{array}{l}\text { Assignation non aléatoire } \\
\text { avec gp contrôle (waitlist) }\end{array}$ & 4 mois & $\begin{array}{l}\text { MdT, fonctionnement exécutif } \\
\text { quotidien, symptômes TDA/H }\end{array}$ \\
\hline $\begin{array}{l}\text { Holmes } \\
\text { et al., } \\
2010\end{array}$ & 25 & 8 à 11 & $\begin{array}{l}\varnothing \text { gp contrôle } \\
\text { Évaluations avec et sans } \\
\text { médication }\end{array}$ & 6 mois & $\begin{array}{l}\text { MdT verbale et visuospatiale, } \\
\text { QI }\end{array}$ \\
\hline $\begin{array}{l}\text { Hovik et } \\
\text { al., } 2013\end{array}$ & 67 & 10 à 12 & $\begin{array}{l}\text { Assignation aléatoire avec } \\
\text { gp contrôle (treatment-as- } \\
\text { usual) }\end{array}$ & 8 mois & MdT verbale et visuospatiale \\
\hline $\begin{array}{l}\text { Van Der } \\
\text { Donk et } \\
\text { al., } 2015\end{array}$ & 102 & 8 à 12 & $\begin{array}{l}\text { Assignation aléatoire avec } \\
\text { gp contrôle actif (Paying } \\
\text { Attention in Class) }\end{array}$ & 6 mois & $\begin{array}{l}\text { MdT, attention, planification, } \\
\text { inhibition, rendement } \\
\text { académique, comportement en } \\
\text { classe, qualité de vie }\end{array}$ \\
\hline $\begin{array}{l}\text { Bigorra et } \\
\text { al., } 2016\end{array}$ & 55 & 7 à 12 & $\begin{array}{l}\text { Assignation aléatoire avec } \\
\text { gp contrôle actif (version } \\
\text { placebo) } \\
\text { Double aveugle }\end{array}$ & 6 mois & $\begin{array}{l}\text { MdT, fonctions exécutives, } \\
\text { rendement académique, } \\
\text { symptômes TDA/H }\end{array}$ \\
\hline
\end{tabular}


Les résultats de l'étude menée par Klingberg et al. (2005) indiquent que les effets du programme Cogmed sur la MdT ainsi que la généralisation des acquis sur l'inhibition et le raisonnement non verbal persistent trois mois après l'intervention. Les effets se maintiennent aussi en ce qui a trait à la réduction des symptômes liés au TDA/H rapportés par les parents. Les résultats de Beck et al. (2010) suggèrent également une amélioration jusqu'à 4 mois postintervention des symptômes associés au TDA/H et du fonctionnement exécutif quotidien, tel que mis en évidence par les réponses des parents aux questionnaires. Ces effets ne sont toutefois pas obtenus auprès des enseignants. Holmes et al. (2010) ont quant à eux comparé les effets du programmes Cogmed à ceux de la médication. Leurs résultats suggèrent que la médication permet d'améliorer la composante visuospatiale de la MdT, alors que l'entrainement cognitif a des effets plus larges et procure des gains sur chaque composante de la MdT. Les auteurs observent un maintien des acquis sur la composante exécutive de la MdT, soit l'administrateur central, six mois après l'intervention. De leur côté, Hovik et al. (2013) ont trouvé que les effets du programme Cogmed sur la MdT auditivo-verbale et visuospatiale persistent jusqu'à huit mois après l'intervention. Ces auteurs relèvent également l'apparition de certains gains sur la composante exécutive de la MdT responsable de la manipulation mentale des informations lors du suivi huit mois post-intervention. Van der Donk et al. (2015) ont quant à eux comparé les effets du programme Cogmed à ceux du programme « Paying attention in class », soit un programme ciblant à la fois la MdT et les fonctions exécutives. Dans l'ensemble, les résultats ne permettent pas de démontrer la supériorité du programme Cogmed par rapport à l'autre intervention, sauf en ce qui a trait à la MdT visuospatiale. Un maintien des acquis sur les mesures améliorées suite à l'entrainement de la MdT est observé six mois post-intervention. Enfin, les résultats de Bigorra et al. (2016) montrent des effets positifs immédiats du programme 
Cogmed sur la MdT, les fonctions exécutives et les symptômes du TDA/H, ainsi que des améliorations tardives lors du suivi six mois post-intervention. Les auteurs insistent sur l'importance du suivi à long terme, lequel s'est avéré nécessaire pour détecter des changements au plan comportemental. Notons que ces deux dernières études n'ont pas été en mesure d'objectiver une amélioration significative du rendement académique, tant à court terme qu'à plus long terme.

Une autre étude a quant à elle examiné les effets à long terme du programme Cogmed auprès de 42 enfants ayant de faibles capacités de MdT, mais n'ayant pas de diagnostic de TDA/H (Holmes, Gathercole et Dunning, 2009). Les résultats indiquent une amélioration significative du groupe expérimental par rapport au groupe contrôle actif sur les habiletés de MdT, ainsi qu'un maintien des acquis à long terme. Notons qu'une amélioration tardive des compétences en mathématique est documentée lors du suivi six mois post-intervention. Des résultats similaires ont été obtenus auprès d'adolescents présentant une déficience intellectuelle (Van der Molen et al., 2010). Ainsi, les effets du programme Cogmed semblent parfois apparaître non pas immédiatement après l'intervention, mais quelques mois plus tard, notamment en raison de la forte influence des apprentissages antérieurs (Gathercole, 2014).

Bien que l'ensemble des études mentionnées précédemment aient mis en évidence un maintien des acquis 3 à 8 mois suivant l'intervention, celles-ci font face à certaines limites méthodologiques compromettant la généralisation des résultats. En effet, deux études seulement compare l'entrainement Cogmed à une version placebo du programme avec des évaluations réalisées en double insu (Bigorra et al., 2016; Klingberg et al., 2005). Deux études comprennent un groupe témoin non actif de type liste d'attente (Beck et al., 2010; Hovik et al., 2013) et une étude ne comprend pas de groupe témoin (Holmes et al., 2010). De plus, la majorité des études 
ne contrôlent pas la prise de médication, alors que son utilisation est commune dans le traitement du TDA/H. Seules les études de Klingberg et al. (2005) et Bigorra et al. (2016) incluent uniquement des participants ne prenant pas de psychostimulants. Enfin, la présentation du TDA/H et la présence de comorbidités ne sont pas systématiquement contrôlées. Notons par ailleurs que l'ensemble des effets objectivés à plus long terme sur les capacités de MdT sont mesurés par des tâches similaires à celles utilisées lors de l'entrainement cognitif et que l'impact pratique d'une telle amélioration reste à démontrer. De plus, les effets indirects à plus long terme du programme Cogmed sur l'attention, l'inhibition, le raisonnement non verbal, les symptômes liés au TDA/H et le rendement scolaire sont variables et demeurent controversés. Ces constats remettent donc en question l'efficacité à plus long terme du programme Cogmed. D'autres études plus rigoureuses sur le plan méthodologique sont nécessaires afin de pallier les limites soulevées précédemment.

Plus récemment, une étude vérifiait les effets du programme Cogmed sur la MdT ainsi que le transfert des effets sur le fonctionnement cognitif, les symptômes liés au TDA/H et le rendement scolaire auprès de jeunes présentant un TDA/H mixte avec comorbidités et recevant un traitement pharmacologique (Dentz, Guay, Gauthier, Romo et Parent, soumis). Cette étude permet donc d'examiner l'impact du programme Cogmed lorsqu'il est intégré à une prise en charge multimodale du TDA/H, tout en tenant compte des caractéristiques des participants. La spécificité de l'échantillon reflèterait davantage la réalité de la pratique clinique et serait un atout important par rapport aux études antérieures. Les participants étaient assignés de façon aléatoire au groupe expérimental ou au groupe contrôle actif, lequel prenait part à la version placebo du programme. Les évaluateurs et les participants étaient aveugles quant à l'assignation des groupes, permettant ainsi de contrôler les biais liés aux attentes de chacun vis-à-vis les 
différentes interventions proposées. Les participants ont été évalués à trois reprises : (T1) six semaines avant le début de l'intervention, (T2) juste avant l'intervention et (T3) six semaines plus tard, une fois l'entrainement terminé. Les résultats de l'étude n'indiquent aucune amélioration significative de la MdT, ni de transfert vers les autres domaines évalués. Ainsi, les participants ayant réalisé l'entrainement de la MdT Cogmed ne se démarquent pas de ceux ayant pris part à la version placebo. Notons toutefois que le taux d'attrition est important $(n=24)$ et constitue une limite majeure à l'interprétation des résultats.

La présente étude s’inscrit en continuité avec celle tout juste décrite de Dentz et al. (soumis). Compte tenu de l'absence d'acquis mis en évidence immédiatement après l'intervention, la présente étude vise à suivre la progression des effets du programme Cogmed afin de vérifier un potentiel effet à retardement six mois post-intervention. Autrement dit, cette étude vise à déterminer si le passage du temps permet de différencier les groupes (expérimental vs contrôle) en favorisant l'apparition de certains effets positifs différés. L’objectif principal consiste plus précisément à évaluer les effets à plus long terme du programme Cogmed sur la MdT auprès de jeunes présentant un TDA/H tout en tenant compte de certaines caractéristiques des participants (présentation mixte du TDA/H, troubles associés, médication). L’objectif secondaire consiste à évaluer le transfert des effets à plus long terme sur l'inhibition, l'attention, le raisonnement non verbal, la compréhension de lecture et le raisonnement mathématique. La perception des parents quant au fonctionnement exécutif quotidien et aux symptômes du TDA/H sera également explorée. 


\section{Méthodologie}

\section{Participants}

Cette étude comprend 31 participants ayant participé à l'étude de Dentz et al. (soumis), âgés entre 7 et 13 ans et présentant un TDA/H de présentation mixte avec troubles associés. Le Tableau 2 détaille les principales caractéristiques de l'échantillon en fonction de chaque groupe. Les diagnostics de TDA/H et troubles associés ont été effectués conformément aux critères du DSM-IV (APA, 2003) par des praticiens de la Clinique des troubles de l'attention de l'Hôpital Rivière-des-Prairies (Québec, Canada) à la suite d'un entretien clinique et de l'analyse de questionnaires comportementaux visant à évaluer les symptômes du TDA/H remplis par les parents et l'enseignant de l'enfant. Pour participer à l'étude, les participants devaient recevoir un traitement pharmacologique stable depuis deux mois et présenter l'un des troubles associés suivants : trouble des apprentissages, trouble du langage, trouble d'opposition avec provocation ou syndrome de Gilles de la Tourette. Les participants devaient également disposer d'un ordinateur et d'une connexion internet au domicile pour avoir accès au programme informatisé Cogmed. Les critères d'exclusion consistaient à avoir un diagnostic d'épilepsie, un trouble internalisé (trouble anxieux ou de l'humeur), un trouble du spectre de l'autisme ou une déficience intellectuelle. Il s'agit d'une étude contrôlée avec assignation aléatoire des participants. Ces derniers ont donc été répartis au hasard à l'un ou l'autre des groupes suivants avant de débuter l'entrainement cognitif:

- Groupe expérimental $(n=30)$ : programme informatisé Cogmed, entrainement cognitif de la mémoire de travail.

- Groupe contrôle actif $(n=30)$ : programme informatisé Cogmed, version de comparaison, entrainement cognitif de la mémoire de travail de faible intensité. 
Parmi les 60 participants ayant initialement accepté de prendre part à l'étude de Dentz et al. (soumis), 36 ont complété l'entrainement et 31 ont pris part à la présente étude visant à évaluer les effets six mois plus tard. Ce taux d'attrition $(n=5)$ s'expliquerait notamment par un manque de motivation de la part de l'enfant ou du parent en lien avec la durée de l'étude, les nombreux déplacements requis, l'absence de compensation financière, la sévérité du tableau clinique, etc.

Tableau 2

Statistiques descriptives des participants

\begin{tabular}{lcc}
\hline & $\begin{array}{c}\text { Groupe expérimental } \\
(\mathbf{n}=\mathbf{1 7})\end{array}$ & $\begin{array}{c}\text { Groupe placebo } \\
(\mathbf{n}=\mathbf{1 4})\end{array}$ \\
\hline Âge moyen & 11 & 9,6 \\
Sexe masculin (\%) & $13(76)$ & $12(86)$ \\
Ethnicité (\%) & & $12(86)$ \\
Caucasien & $16(94)$ & $2(14)$ \\
Autre & $1(6)$ & $14(100)$ \\
TDAH mixte (\%) & $17(100)$ & $6(43)$ \\
Comorbidités (\%) & & $2(14)$ \\
Trouble d'opposition avec provocation & $5(29)$ & $2(14)$ \\
Trouble de langage & $3(18)$ & $6(43)$ \\
Trouble des apprentissages & $7(41)$ & \\
Syndrome de Gilles de la Tourette & $9(53)$ & $10(71)$ \\
Médication (\%) & & $1(7)$ \\
Psychostimulants à base d'amphétamines & $9(53)$ & $7(50)$ \\
Psychostimulants à base de méthylphénidate & $7(41)$ & $7(41)$ \\
Non psychostimulants & $6(35)$ & \\
Antipsychotiques & & \\
\hline
\end{tabular}




\section{Procédures}

Cette étude a reçu l'approbation du comité d'éthique de l'Hôpital Rivière-des-Prairies (2017). L'évaluation cognitive a eu lieu six mois post-intervention (T4) et s'est déroulée dans les mêmes conditions que celles effectuées préalablement dans le cadre de l'étude de Dentz et al. (soumis), c'est-à-dire sous médication, avec les mêmes instruments de mesure et selon un devis en double aveugle. Le parent et l'enseignant étaient invités à remplir à nouveau les mêmes questionnaires. Notons toutefois qu'un nombre insuffisant de questionnaires fut remplis par les enseignants pour permettre d'analyser ces données.

\section{Instruments de mesure}

Les instruments de mesure en lien avec l'évaluation cognitive seront d'abord présentés, suivis des questionnaires remis aux parents.

Mémoire de travail auditivo-verbale. La MdT auditivo-verbale a été mesurée par les sous-tests Séquences de chiffres et Séquences lettres-chiffres de l'Échelle d'intelligence de Wechsler pour enfants, $4^{\mathrm{e}}$ édition (WISC-IV; Wechsler, 2005a). La première épreuve consiste à retenir une suite de chiffres présentés oralement, dont la longueur augmente graduellement. Le participant doit répéter la série de chiffres dans l'ordre direct, puis dans l'ordre inverse afin de solliciter sa MdT verbale. Le coefficient de cohérence interne de ce test est satisfaisant $(\alpha=$ $0,84)$, tout comme la fidélité test-retest $(r=0,84)$. La deuxième épreuve consiste quant à elle à retenir une séquence de chiffres et de lettres présentés oralement, dont la longueur augmente graduellement. Le participant doit d'abord répéter les chiffres, en ordre croissant, puis les lettres, en ordre alphabétique. Le coefficient de cohérence interne $(\alpha=0,90)$ et l'indice de fidélité testretest $(r=0,76)$ sont satisfaisants.

Mémoire de travail visuospatiale. La MdT visuospatiale a été mesurée par le sous-test 
Empan spatial de l'Échelle non verbale d'intelligence de Wechsler ( $W N V$; Wechsler, 2006). Une version informatisée de cette tâche a été utilisée afin d'uniformiser le rythme de présentation des stimuli. Cette tâche consiste à retenir l'ordre dans lequel une série de carrés disposés à l'écran se colorent de bleu successivement. Le participant doit reproduire la série proposée dans le même ordre ou dans l'ordre inverse, selon la consigne. Les coefficients de cohérence interne de la version originale sont adéquats, avec des valeurs allant de $\alpha=0,79$ (ordre direct) à $\alpha=0,87$ (score total). La fidélité test-retest est satisfaisante $(r=0,79)$.

Inhibition et attention. L'inhibition et l'attention ont été évaluées à partir du Test de performance continue $-2^{\mathrm{e}}$ édition (CPT-II ; Conners, 2000). Ce test consiste à appuyer le plus rapidement possible sur la barre d'espacement du clavier d'ordinateur lorsqu'une lettre apparaît à l'écran, sauf pour la lettre X. Le CPT-II dure 14 minutes et présente 360 stimuli, avec des intervalles inter-stimuli de 1, 2 ou 4 secondes. Les mesures suivantes du CPT-II ont été retenues : erreurs de commission et erreurs d'omission. Les erreurs de commission indiquent généralement la présence d'impulsivité, alors que les erreurs d'omission traduisent généralement la présence d'inattention. Les qualités psychométriques du CPT-II sont adéquates, avec des indices de fidélité test-retest qui varient entre $r=0,43$ et $r=0,89$ (Conners et Sitarenios, 2011). Le CPT-II présente également une bonne sensibilité et spécificité envers le TDA/H chez des enfants de 6 à 17 ans, avec des indices de $83 \%$ et $82 \%$ respectivement (Conners et Sitarenios, 2011).

Raisonnement non verbal. Le raisonnement non verbal a été mesuré par le test des Matrices colorées progressives de Raven (CPM; Raven et Court, 1998). Ce test comprend 36 problèmes répartis en trois sections de 12 problèmes de difficulté croissante. Le participant observe une matrice incomplète et doit sélectionner la partie manquante parmi des choix de 
réponses. Le coefficient de cohérence interne $(\alpha=0,80-0,94)$ ainsi que l'indice de fidélité testretest $(r=0,87)$ sont satisfaisants.

Compréhension de lecture et raisonnement mathématique. Les mesures du rendement scolaire incluaient les sous-tests Compréhension de lecture et Raisonnement mathématique du Test de rendement individuel de Wechsler, $2^{\mathrm{e}}$ édition, version pour francophones (WIAT-II CDN$F$; Wechsler, 2005b). La première épreuve évalue différentes compétences en compréhension de lecture telles que lire des textes et répondre à des questions sur le contenu. La deuxième épreuve évalue quant à elle différentes compétences en raisonnement mathématique telles que compter, identifier des formes géométriques, résoudre des problèmes simples ou complexes, interpréter des graphiques, etc. La WIAT-II présente de bonnes propriétés psychométriques en termes de validité. Les épreuves de lecture et de mathématique présentent d'excellents coefficients de fidélité, soit $r=0,95$ et $r=0,94$.

Fonctions exécutives au quotidien. Les fonctions exécutives au quotidien ont été mesurées par l'Inventaire des comportements reliés aux fonctions exécutives, version parent (BRIEF ; Gioia, Isquith, Guy et Kenworth, 2000). Ce questionnaire comprend 86 items au total, lesquels sont évalués selon une échelle de type Likert de 0 à 2 ( $0=$ Jamais ou rarement ; 1=Quelques fois ; 2=Souvent). Les qualités psychométriques du questionnaire BRIEF sont jugées adéquates. En effet, le coefficient de cohérence interne varie entre 0,80 et 0,98 et l'indice de fidélité test-retest est de 0,81 (Gioia et al., 2000). La validité de construit du BRIEF serait également acceptable, tel qu'en témoignent les corrélations avec d'autres questionnaires similaires (Gioia et al., 2000). L'échelle globale des fonctions exécutives a été retenue pour examiner les comportements reliés aux fonctions exécutives dans différents milieux (c.-à-d., inhibition, flexibilité, contrôle émotionnel, autorégulation, planification/ organisation, MdT, 
initiative, organisation du matériel). Cette échelle reflèterait assez bien le fonctionnement de l'enfant au quotidien.

Symptômes liés au TDA/H. La symptomatologie liée au TDA/H a été évaluée à l'aide du questionnaire Conners $3^{\mathrm{e}}$ édition (Conners, 2008). La version longue (99 items) destinée aux parents pour les jeunes de 6 à 18 ans a été utilisée. Ce questionnaire se base sur les manifestations comportementales du TDA/H telles que décrites dans le DSM-IV et s'intéresse au comportement de l'enfant durant le dernier mois, selon une échelle de type likert de 0 à $3(0=$ Jamais ou rarement ; $1=$ Quelques fois $; 2=$ Souvent et $3=$ Très souvent). La consistance interne du Conners 3 - version parent est adéquate, avec un alpha de Cronbach de 0,91 (Kao et Thomas, 2010). Les sous-échelles inattention et hyperactivité/impulsivité ont été retenues. Le coefficient moyen de fiabilité pour les échelles associées aux symptômes du DSM-IV est très satisfaisant ( $\alpha$ $=0,90)$, tout comme la validité de construit (Kao et Thomas, 2010). La stabilité temporelle et la fidélité inter-juges pour les échelles retenues sont acceptables, avec des coefficients de 0,89 et 0,84 respectivement (Kao et Thomas, 2010).

\section{Résultats}

Pour répondre à l'objectif de la présente étude, les deux groupes (expérimental et contrôle actif) ont été comparés entre le deuxième et le quatrième temps de mesure par une série d'analyses de variance (ANOVA) à plan mixte $(2 \times 2)$ réalisées sur chacune des variables dépendantes examinées. Cette analyse a pour but de vérifier l'évolution des capacités de chaque groupe à travers le temps, c'est-à-dire entre le pré-test effectué au début de l'intervention (T2) et le suivi six mois post-intervention (T4). Rappelons que le premier temps de mesure (T1) réalisé dans le cadre de l'étude de Dentz et al. (soumis) avait lieu six semaines avant le début de l'intervention afin de contrôler l'évolution spontanée des participants. 
Puisqu'aucun acquis en lien avec la réalisation du programme Cogmed n'a été mis en évidence directement après l'intervention, une amélioration du groupe expérimental par rapport au groupe témoin entre $\mathrm{T} 2$ et $\mathrm{T} 4$ représenterait un effet positif à retardement potentiellement attribuable à l'entrainement cognitif, lequel serait mis en évidence par un effet d'interaction statistiquement significatif. Les effets d'interaction ont donc été testés. Lorsqu'il n'y avait pas d'interaction, les effets principaux étaient alors interprétés. Un seuil de signification de 5\% a été utilisé $\left(\alpha_{\text {critique }}=.05\right)$ pour l'ensemble des analyses effectuées.

Compte tenu du faible échantillon, les participants ayant des scores extrêmes ont été conservés. Les trois scores extrêmes repérés ont été remplacés par des scores situés à la limite de $\mathrm{z}=+/-3.29$ (Tabachnick et Fidell, 2007). Les quelques données manquantes ont quant à elles été remplacées par une estimation découlant de l'algorithme EM (Expectation-Maximization). La distribution de chaque variable dépendante a été inspectée selon les critères de Curran, West et Finch (1996) afin de s'assurer de la normalité des distributions. Les indices d'asymétrie (skewness) et de voussure (kurtosis) étaient généralement compris entre +/- 2 et +/- 7 , respectivement. L'ANOVA étant relativement robuste aux écarts de la normalité, il est possible de procéder aux analyses sans avoir une normalité parfaite (Field, 2013).

Le Tableau 3 comprend un résumé des principales statistiques examinées dans le cadre des analyses. Les scores pondérés ont été utilisés pour les mesures du WISC-IV, du WNV et du WIAT-II. Les scores t ont été utilisés pour les questionnaires (Conners, BRIEF) et pour les mesures du CPT-II. Les scores bruts ont été utilisés pour les Matrices de Raven (CPM) puisque les normes disponibles allaient de 5 à 11 ans uniquement. 
Tableau 3

Résultats de l'ANOVA mixte - effets d'interaction et effets principaux

\begin{tabular}{|c|c|c|c|c|c|c|c|c|c|c|c|c|c|c|}
\hline & \multicolumn{2}{|c|}{ Expérimental } & \multicolumn{2}{|c|}{ Placebo } & \multicolumn{2}{|c|}{ Expérimental } & \multicolumn{2}{|c|}{ Placebo } & \multirow[b]{3}{*}{$F_{T x G}$} & \multirow[b]{3}{*}{$P_{T x G}$} & \multirow[b]{3}{*}{$F_{T}$} & \multirow[b]{3}{*}{$P_{T}$} & \multirow[b]{3}{*}{$F_{G}$} & \multirow[b]{3}{*}{$P_{G}$} \\
\hline & $\mathrm{T} 2$ & $\mathrm{~T} 2$ & $\mathrm{~T} 2$ & $\mathrm{~T} 2$ & $\mathrm{~T} 4$ & $\mathrm{~T} 4$ & $\mathrm{~T} 4$ & T4 & & & & & & \\
\hline & $M$ & $E T$ & $M$ & $E T$ & $M$ & $E T$ & $M$ & $E T$ & & & & & & \\
\hline \multicolumn{15}{|l|}{ WISC-IV } \\
\hline $\mathrm{SC}$ & 8.41 & 1.50 & 8.50 & 2.50 & 10.06 & 1.78 & 9.93 & 3.69 & 0.07 & .792 & 14.44 & .001 & 0.00 & .979 \\
\hline - Direct & 7.24 & 0.90 & 6.82 & 1.71 & 8.59 & 1.33 & 7.50 & 2.03 & 2.32 & .139 & 22.62 & .000 & 2.27 & .143 \\
\hline - Indirect & 6.00 & 1.23 & 5.36 & 1.45 & 7.00 & 1.94 & 6.64 & 2.24 & 0.24 & .626 & 15.31 & .001 & 0.80 & .378 \\
\hline SLC & 9.18 & 2.27 & 9.43 & 2.38 & 9.06 & 1.44 & 8.50 & 2.59 & 1.06 & .313 & 1.52 & .228 & 0.05 & .824 \\
\hline \multicolumn{15}{|l|}{ WNV } \\
\hline Empan spatial & 12.82 & 4.33 & 11.36 & 5.03 & 14.76 & 3.85 & 13.86 & 4.35 & 0.15 & .706 & 9.04 & .005 & 0.72 & .404 \\
\hline - Direct & 9.88 & 3.28 & 8.86 & 3.21 & 10.65 & 2.94 & 10.79 & 3.22 & 1.25 & .273 & 6.19 & .019 & 0.19 & .665 \\
\hline - Indirect & 9.59 & 3.39 & 9.93 & 4.07 & 10.76 & 2.97 & 11.07 & 2.62 & 0.00 & .984 & 2.08 & .160 & 0.14 & .713 \\
\hline \multicolumn{15}{|l|}{ CPM } \\
\hline Matrices & 32.53 & 3.24 & 29.57 & 5.53 & 33.06 & 2.56 & 31.47 & 5.49 & 3.75 & .063 & 10.64 & .003 & 2.29 & .141 \\
\hline \multicolumn{15}{|l|}{ CPT-II } \\
\hline Commissions & 49.92 & 12.17 & 51.44 & 12.43 & 49.15 & 13.48 & 52.51 & 11.88 & 0.20 & .655 & 0.00 & .977 & 0.37 & .550 \\
\hline Omissions & 54.65 & 17.09 & 53.47 & 12.84 & 50.40 & 16.31 & 56.39 & 17.64 & 1.94 & .175 & 0.16 & .697 & 0.21 & .649 \\
\hline \multicolumn{15}{|l|}{ WIAT-II } \\
\hline Lecture & 105.24 & 16.82 & 95.29 & 23.94 & 107.06 & 12.08 & 95.79 & 20.15 & 0.14 & .709 & 0.49 & .488 & 2.75 & .108 \\
\hline Mathématique & 100.18 & 12.13 & 101.93 & 15.87 & 95.12 & 13.56 & 98.57 & 19.49 & 0.25 & .619 & 6.47 & .017 & 0.25 & .623 \\
\hline \multicolumn{15}{|c|}{ Conners3 - DSM-IV } \\
\hline Inattention & 71.94 & 13.26 & 76.21 & 9.35 & 72.90 & 10.47 & 72.10 & 8.21 & 2.58 & .119 & 0.71 & .406 & 0.24 & .625 \\
\hline $\begin{array}{l}\text { Hyperactivité } \\
\text { /Impulsivité }\end{array}$ & 72.59 & 11.54 & 76.53 & 10.33 & 74.98 & 15.32 & 74.41 & 14.38 & 1.46 & .238 & 0.04 & .851 & 0.15 & .702 \\
\hline BRIEF & & & & & & & & & & & & & & \\
\hline ÉGFE & 65.18 & 10.02 & 67.43 & 8.88 & 64.67 & 11.50 & 68.36 & 8.21 & 0.39 & .537 & 0.02 & .904 & 0.78 & .384 \\
\hline
\end{tabular}

Note . Expérimental $=$ groupe ayant pris part au programme Cogmed $(\mathrm{n}=17) ;$ Placebo $=$ groupe contrôle actif $(\mathrm{n}=$ 14) ; $\mathrm{T} 2=$ Temps de mesure 2 (pré-intervention) ; $\mathrm{T} 4=$ Temps de mesure 4 (six mois post-intervention) ; $\mathrm{SC}=$ Séquences de chiffres; SLC $=$ Séquences lettres-chiffres; ÉGFE = Échelle globale des fonctions exécutives; $M=$ moyenne ; $E T$ = écart-type; $F_{T x G}=$ statistique $\mathrm{F}$ associée à l'effet d'interaction temps* groupe; $P_{T x G}=$ effet d'interaction temps*groupe; $F_{T}=$ statistique $\mathrm{F}$ associée à l'effet principal du temps; $P_{T}=$ effet principal du temps; $F_{G}=$ statistique $\mathrm{F}$ associée à l'effet principal du groupe; $P_{G}=$ effet principal du groupe 


\section{Mémoire de travail}

Les résultats n'indiquent aucun effet d'interaction (temps x groupe) significatif, et ce, tant au niveau de la MdT auditivo-verbale qu'au niveau de la MdT visuospatiale. Ainsi, les participants du groupe expérimental ne diffèrent pas de façon statistiquement significative du groupe placebo à travers le temps sur ces variables. Les scores globaux, tout comme les scores associés aux empans directs et indirects ont été examinés pour ces deux variables.

Les résultats révèlent toutefois un effet principal du temps statistiquement significatif en ce qui a trait à l'empan de chiffres (WISC-IV - Séquences de chiffres), tant direct qu'indirect, reflétant une amélioration significative de la MdT auditivo-verbale entre T2 et T4. En effet, le score total moyen augmente de façon significative entre le début de l'entrainement cognitif $(M=$ $8,45)$ et le suivi effectué six mois plus tard $(M=10,00)$, indépendamment du groupe d'appartenance. La taille d'effet indique un grand effet $\left(\eta^{2}=0,332\right)$.

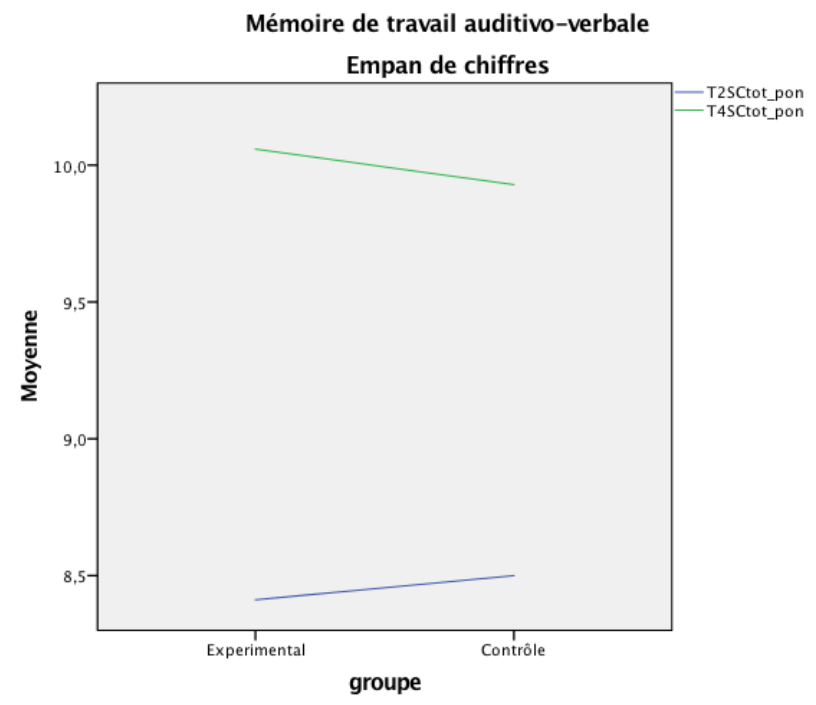

Figure 1. Effet principal du temps pour la mémoire de travail auditivo-verbale 
Les résultats montrent également un effet principal du temps significatif en ce qui concerne l'empan spatial, reflétant ainsi une amélioration des capacités de MdT visuospatiale entre T2 et T4. En effet, la différence des moyennes entre le début de l'entrainement cognitif ( $M$ $=12,16)$ et le suivi effectué six mois plus tard $(M=14,35)$, indique une amélioration significative de la performance, et ce, indépendamment du groupe d'appartenance. La taille d'effet indique à nouveau un grand effet $\left(\eta^{2}=0,238\right)$. Notons cependant que seul l'empan spatial direct connaît une amélioration significative.

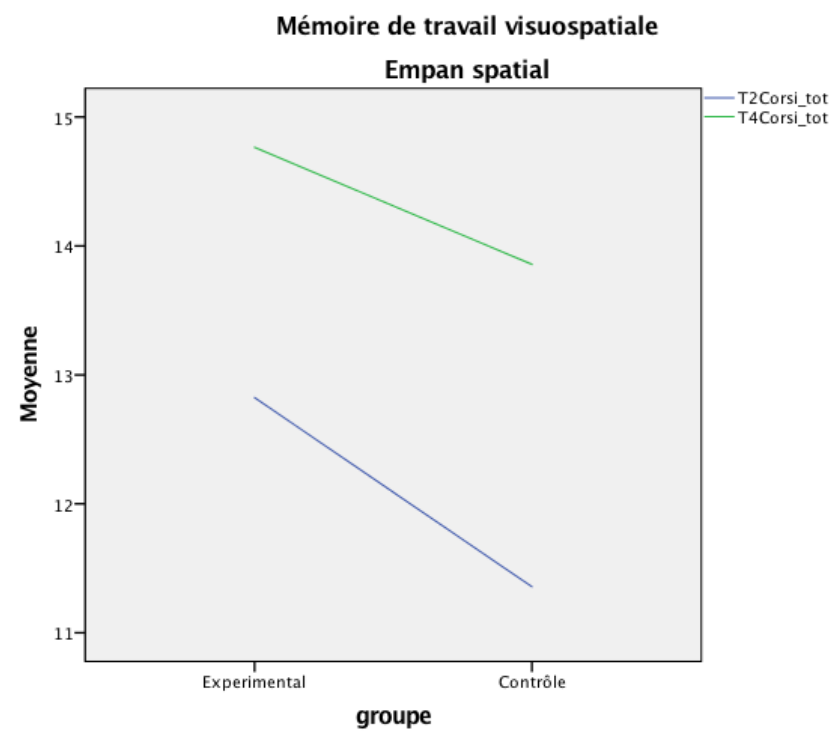

Figure 2. Effet principal du temps pour la mémoire de travail visuospatiale

\section{Raisonnement non verbal, fonctions attentionnelles et inhibition}

En ce qui concerne le transfert des effets vers les capacités d'attention, d'inhibition et de raisonnement non-verbal, les résultats ne mettent en évidence aucun effet d'interaction (temps $\mathrm{x}$ groupe) significatif. Les participants ayant pris part au programme Cogmed n'améliorent pas davantage leur performance au CPT-II et au CPM, comparativement aux participants ayant pris part à la version placebo du programme. 
Les résultats montrent cependant un effet principal du temps statistiquement significatif en ce qui a trait au raisonnement non verbal. En effet, la moyenne du score brut total associé à la tâche des Matrices colorées progressives de Raven augmente de façon significative entre le début de l'entrainement $(M=31,19)$ et le suivi six mois plus tard $(M=32,34)$, indifféremment du groupe d'appartenance. La taille d'effet suggère un effet de grande taille $\left(\eta^{2}=0,268\right)$.

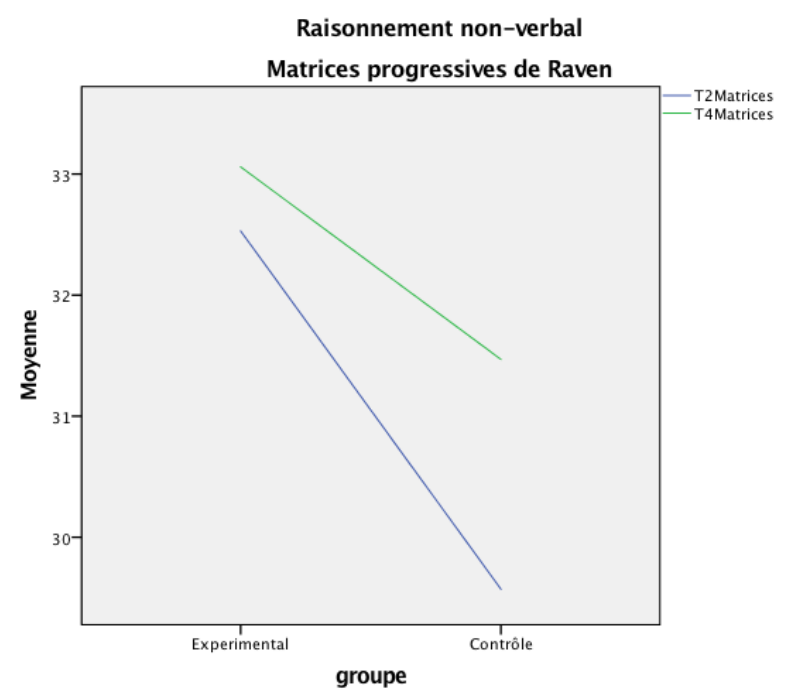

Figure 3. Effet principal du temps pour le raisonnement non verbal

\section{Fonctionnement exécutif quotidien et symptômes associés au TDA/H}

L'analyse des effets à long terme du programme Cogmed sur l'échelle globale des fonctions exécutives et les symptômes liés au TDA/H ne montre aucun effet bénéfique six mois après la fin du traitement. En effet, aucun effet d'interaction significatif n'est mis évidence sur les mesures subjectives liées au fonctionnement exécutif quotidien ainsi qu'aux comportements d'inattention et d'hyperactivité/ impulsivité. Aucun effet principal n'est obtenu par rapport à ces variables. Le programme Cogmed n'a donc pas d'effets différés sur les problèmes de comportements associés aux fonctions exécutives de même que sur l'ampleur des symptômes comportementaux perçus par les parents. 


\section{Rendement scolaire}

Les résultats de l'ANOVA mixte ne montrent pas d'effet d'interaction significatif en ce qui concerne les capacités de compréhension de lecture et de raisonnement mathématique. Les participants ayant pris part au programme Cogmed n'améliorent pas davantage leur performance aux tâches de rendement scolaire du WIAT-II, comparativement aux participants ayant pris part à la version placebo du programme.

Les effets principaux ne sont pas significatifs en ce qui a trait aux capacités de compréhension de lecture. Les résultats indiquent cependant un effet principal du temps significatif en ce qui a trait au raisonnement mathématique. En effet, nous observons une diminution significative des performances entre la fin de l'entrainement $(M=100,97)$ et le suivi six mois plus tard $(M=96,68)$, tous groupes confondus. La taille d'effet révèle un grand effet $\left(\eta^{2}\right.$ $=0,182)$.

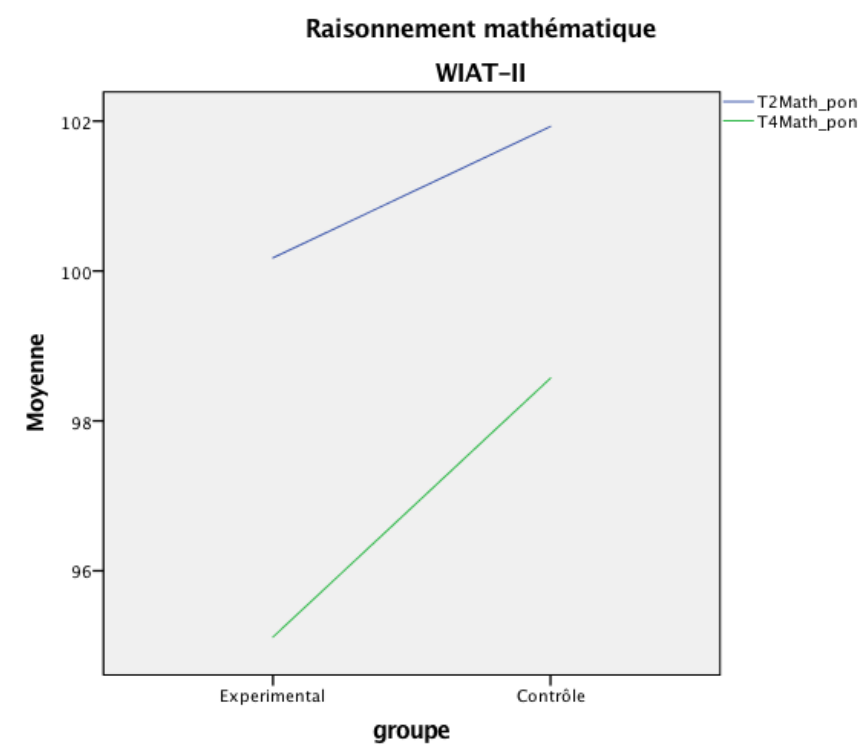

Figure 4. Effet principal du temps pour le raisonnement mathématique 


\section{Discussion}

L’objectif principal de la présente étude était d'examiner les effets à long terme du programme Cogmed sur la MdT auprès d'enfants présentant un TDA/H mixte avec comorbidités et bénéficiant d'un traitement pharmacologique. Le transfert des effets à plus long terme sur des mesures secondaires non ciblées par l'entrainement, soit l'attention, l'inhibition, le raisonnement non verbal, la compréhension de lecture, le raisonnement mathématique, le fonctionnement exécutif quotidien et les symptômes liés au TDA/H était également étudié à titre exploratoire.

Le passage du temps ne semble pas favoriser l'apparition d'acquis chez les jeunes ayant profité du programme Cogmed. En effet, les résultats de l'étude ne montrent aucune amélioration six mois post-intervention pour les jeunes ayant bénéficié du programme Cogmed en comparaison aux jeunes ayant pris part à la version placebo du programme en ce qui concerne la MdT auditivo-verbale et visuospatiale. De plus, aucun effet de transfert sur les capacités d'attention, d'inhibition, de raisonnement non verbal, de compréhension de lecture et de raisonnement mathématique n'émerge à travers le temps pour le groupe expérimental, comparativement au groupe contrôle actif. Enfin, la réalisation du programme Cogmed ne permet pas à plus long terme de distinguer les groupes en ce qui a trait à aux symptômes associés au TDA/H et aux problèmes exécutifs au quotidien, tels qu'évalués par les parents. La présente étude n'est donc pas en mesure de confirmer les bienfaits du programme Cogmed à plus long terme lorsque l'entrainement cognitif est réalisé de manière complémentaire à un traitement pharmacologique auprès de jeunes ayant un TDA/H mixte avec comorbidité(s). Ces résultats vont dans le même sens qu'une étude pilote récente ayant investigué l'impact additionnel d'un programme d'entrainement cognitif (ACTIVATE) par rapport à la médication (Rosa et al., 2017). Leurs résultats, bien que préliminaires, montrent que le groupe expérimental s'améliore autant 
que le groupe contrôle. L'entrainement cognitif, lorsqu'utilisé comme adjuvant à la médication, ne permet pas de distinguer les groupes et ne semble donc pas avoir d'impact sur le plan clinique. De tels résultats interrogent donc sur la pertinence d'utiliser l'entrainement cognitif en guise de traitement complémentaire pour le TDA/H.

Par ailleurs, des effets principaux du temps traduisant une amélioration significative de la performance ont été obtenus à l'endroit de la MdT auditivo-verbale, de la MdT visuospatiale et du raisonnement non verbal. Si une amélioration est notée sur ces mesures entre le prétest et le suivi six mois post-intervention, le groupe expérimental ne se distingue pas du groupe placebo. Ainsi, nous ne pouvons attribuer ces acquis différés au fait d'avoir pris part au programme Cogmed. Ces effets principaux pourraient entre autres s'expliquer par un effet test-retest et/ou par un effet de maturation du cerveau lié au passage du temps. Ceux-ci pourraient également refléter des effets non spécifiques induits par le contexte relationnel entre le sujet, le parent et le clinicien (Van der Dunk et al., 2016). Les parents auraient en effet tendance à percevoir positivement l'efficacité du programme Cogmed, ce qui pourrait influencer leur façon d'interagir avec l'enfant (Graham et Benninger, 2016).

Un effet principal du temps traduisant une diminution de la performance à une tâche de raisonnement mathématique a aussi été obtenu. Bien que cette détérioration soit significative, celle-ci s'applique de façon indifférenciée aux deux groupes. Cet effet principal pourrait notamment s'expliquer par le changement d'évaluatrice et/ou le changement d'année scolaire entre les deux temps de mesure impliqués. Les normes de cotation de même que la composition du test ont ainsi subi certains ajustements en lien avec l'année et le trimestre scolaires (p. ex., questions plus difficiles). Les résultats ne sont donc pas nécessairement comparables d'une évaluation à l'autre sur cette mesure particulière. De plus, bien que cette diminution soit 
significative sur le plan statistique, elle ne l'est pas sur le plan clinique puisque les résultats demeurent dans la moyenne aux deux temps de mesure.

Les résultats objectivés dans la présente étude contrastent avec ceux obtenus par d'autres auteurs qui avaient relevé l'apparition ou le maintien d'acquis à plus long terme (Beck et al., 2010; Bigorra et al., 2016; Holmes et al., 2010; Hovik et al., 2013 ; Klingberg et al., 2005; van der Donk et al., 2015). Plusieurs hypothèses peuvent être avancées pour tenter d'expliquer cette divergence. En outre, les résultats pourraient s'expliquer par la prise de médication pendant l'entrainement cognitif et les évaluations. En effet, les résultats de Klingberg et al. (2005), lesquels avaient mis en lumière un maintien des acquis trois mois post-intervention auprès d'enfants présentant un TDA/H sans traitement pharmacologique, ne sont pas reproduits lorsque l'entrainement de la MdT est réalisé en concomitance avec la médication. Or, la majorité des études sur le programme Cogmed et le TDA/H ne contrôlent pas la prise de médication, de sorte qu'il est difficile de distinguer l'apport du programme Cogmed sur l'amélioration des fonctions cognitives par rapport à celui du traitement pharmacologique (Chacko et al., 2013; Van der Donk et al., 2016). Les résultats de la présente étude sont donc difficilement comparables à ceux des études antérieures examinant les effets au long cours du programme Cogmed auprès d'une population de jeunes présentant un TDA/H. Les mesures du niveau de base réalisées préalablement par Dentz et al. (soumis) indiquaient que les habiletés de MdT des participants se situaient dans la moyenne avant même d'entamer l'entrainement cognitif. Il se peut ainsi que les habiletés de MdT aient déjà profité du traitement pharmacologique et que l'effet de la médication ait été suffisant à lui seul pour maintenir cette amélioration dans le temps (Strand et al., 2012). La prise de médication aurait pu normaliser leur performance aux épreuves cognitives, limitant du même coup les chances de détecter une amélioration différée de la MdT. 
Outre la prise de médication, plusieurs études ne tiennent pas compte de la présentation du TDA/H et des comorbidités associées, alors que les effets du programme Cogmed pourraient être différents selon ces critères (Shinaver et al., 2014 ; Van der Donk et al., 2016). Selon Van der Dunk et ses collaborateurs (2016), le programme Cogmed serait plus efficace dans le cas du $\mathrm{TDA} / \mathrm{H}$ à prédominance inattentive que dans le cas du TDA/H à prédominance hyperactive/ impulsive ou mixte. Ces deux présentations ne seraient pas associées au même profil cognitif (Ahmadi, Mohammadi, Araghi et Zarafshan, 2014). La présence de comorbidités ou de déficits plus sévères pourrait également limiter les effets du programme Cogmed (Chacko et al., 2013) et ainsi nécessiter un entrainement prolongé (Shinaver et al., 2014). Les effets d'un entrainement cognitif dont la durée serait plus longue ne sont toutefois pas connus à ce jour, le protocole standard associé au programme Cogmed étant de cinq séances d'environ 40 minutes par semaine, durant cinq semaines consécutives (Shipstead et al., 2012). La majorité des études sur le programme Cogmed comprend en effet un nombre total de 20 à 25 séances réparties sur cinq à six semaines (Rapport et al., 2013). Enfin, peu d'études comprennent des évaluations en double insu ainsi qu'un groupe contrôle actif permettant de contrôler l'effet placebo lié aux attentes des participants et des parents par rapport à la prise en charge. Ces biais méthodologiques limitent grandement l'interprétation des résultats et pourraient expliquer la divergence des résultats obtenus entre la présente étude et d'autres études antérieures. Avec une méthodologie plus rigoureuse et un échantillon plus spécifique, le programme Cogmed procure moins d'effets. Ce constat remet ainsi en question la promotion de ce type d'approche dans la prise en charge multimodale des jeunes ayant un TDA/H mixte avec troubles associés. 


\section{Limites de l'étude}

En raison du taux d'attrition important entre le début de l'entrainement cognitif et le suivi à long terme, la taille de l'échantillon est restreinte. Ce faible effectif limite la puissance statistique et la généralisation des résultats (Deforge, 2011). Outre l'étude de Holmes et al. (2010) qui comprend un échantillon de 25 participants, les études incluant un suivi à plus long terme comprennent en moyenne 64 participants (voir Tableau 1). De fait, un effectif minimum de 30 sujets par groupe est généralement jugé nécessaire pour obtenir suffisamment de puissance statistique (Deforge, 2011). La durée de l'étude, les nombreuses évaluations requises et l'absence de compensation financière ont été avancés comme facteurs potentiels d'attrition. De plus, le profil clinique complexe des participants ainsi que les problèmes de motivation associés au TDA/H ont probablement interféré avec l'adhérence au traitement. En effet, le TDA/H s'accompagne généralement d'un défaut motivationnel et d'une faible tolérance à l'effort mental soutenu (Sonuga-Barke et Halperin, 2010). Or, il faut rappeler que cette étude était plutôt contraignante en termes de temps et qu'un investissement important était requis des jeunes comme des parents. Compte-tenu du niveau d'exigences élevé de l'étude et du délai de six mois entre la fin de l'entrainement et le suivi à long terme, il est probable que le manque de motivation ait joué un rôle majeur dans le taux d'attrition.

Une autre limite de l'étude pourrait être liée à la qualité de l'entrainement cognitif, tel qu'en témoignent différents indices extraits du programme Cogmed. En effet, l'indice de progrès doit être supérieur à 17 pour être considéré acceptable (Chacko et al., 2014; Gray et al., 2012 ; Stevens et al., 2015). Or, à peine 52\% des participants de l'étude de Dentz et al. (soumis) ont atteint ce score, alors que la prévalence était de $70 \%$ et $89 \%$ dans les études respectives de Gray et al. (2012) et Chacko et al. (2014). Ces dernières études montraient quant à elles des effets 
significatifs sur la MdT. Dentz et ses collaborateurs (soumis) ont également mis en lumière une limite en ce qui a trait au temps actif d'entrainement. En effet, la durée moyenne des séances d'entrainement était d'environ 33 minutes, ce qui est significativement plus faible que celles rapportées dans les études antérieures (39-45 minutes; Chacko et al., 2014; Gray et al., 2012; Klingberg et al., 2005; Van der Donk et al., 2015). Cet écart significatif pourrait être lié au manque de motivation soulevé précédemment et expliquer en partie les résultats actuels.

\section{Conclusion}

De plus en plus d'études remettent en question l'efficacité du programme Cogmed, du moins du point de vue de son utilité clinique (Chacko et al., 2013; Cortese et al., 2015; Dentz et al., 2016; Melby-Lervåg et Hulme, 2013; Morrison et Chein, 2011; Rapport et al., 2013; Shipstead et al., 2012; Sonuga-Barke et al., 2013). En effet, si certaines études montrent des effets positifs sur les capacités de MdT ou autres fonctions cognitives, l'impact réel du programme Cogmed sur le fonctionnement quotidien et les symptômes liés au TDA/H reste à démontrer, tout comme le maintien des acquis à plus long terme. Compte-tenu des coûts élevés reliés au programme et du niveau d'engagement requis par le jeune et les parents, l'impact pratique doit être suffisamment important pour justifier la mise en œuvre d'une telle approche dans la prise en charge du TDA/H. La valeur clinique de l'entrainement cognitif est d'autant plus questionnable lorsqu'il est réalisé en concomitance avec un traitement pharmacologique (Van der Oord et al., 2008). En effet, les résultats de la présente étude, en continuité avec celle de Dentz et al. (soumis), montrent que l'entrainement cognitif de la MdT par le programme informatisé Cogmed ne permet pas de bonifier les effets de la médication en favorisant l'apparition d'acquis, tant à court terme qu'à plus long terme. De tels résultats ne permettent pas 
de recommander le programme Cogmed comme approche complémentaire à la médication auprès de jeunes ayant un TDA/H mixte avec comorbidités.

La prise de médication semble influencer les capacités de MdT des participants, lesquels ne présentent pas systématiquement un déficit de la MdT avant d'entreprendre l'entrainement cognitif. La mise en évidence d'un déficit de la MdT préalablement à l'entrainement cognitif semble être un prérequis valable et devrait être examinée dans les futures études. Les effets du programme Cogmed devraient également être étudiés auprès d'échantillons plus homogènes où la prise de médication, la présentation du TDA/H et les comorbidités seraient contrôlées. En effet, il apparait souhaitable de documenter comment les effets du programme Cogmed varient en fonction des caractéristiques des participants. Ce type d'approche pourrait permettre de cibler une clientèle plus spécifique et ainsi optimiser les effets du programme Cogmed.

Certains aménagements au sein du programme pourraient également augmenter l'efficacité de cette intervention et mériteraient d'être explorés dans de futures études. Par exemple, il pourrait être pertinent que les exercices ciblent davantage l'administrateur central, soit la principale composante de la MdT atteinte dans le contexte d'un TDA/H (Kasper et al., 2012). L'entrainement intensif de cette composante, qui relève davantage des fonctions exécutives, pourrait permettre aux jeunes d'atteindre un meilleur contrôle attentionnel, ce qui aurait sans doute de plus larges répercussions sur le fonctionnement cognitif et adaptatif. L'entrainement cognitif pourrait aussi cibler un spectre plus large de fonctions cognitives, notamment sur les plans attentionnel et exécutif (Dentz et al., 2016). Par exemple, le programme Captain's Log Software System comprend 36 exercices répartis en 50 programmes (Farias et al., 2017) et le programme ACTIVATE comprend six jeux ciblant la mémoire de travail, la vitesse de traitement de l'information, l'attention soutenue, l'attention divisée, le raisonnement et 
l'inhibition (Rosa et al., 2017). Ce type de programmes permettrait de tenir compte de l'hétérogénéité des profils cognitifs associés au TDA/H et d'offrir une prise en charge individualisée. Certains auteurs proposent également de varier l'intensité de l'entrainement cognitif en fonction de la sévérité du tableau clinique et des déficits (Shinaver et al., 2014). Les jeunes ayant un TDA/H avec troubles concomitants pourraient entre autres avoir besoin d'un entrainement plus extensif pour répondre à leurs besoins thérapeutiques. D'autres études sont cependant nécessaires pour vérifier si le programme Cogmed est avantageux lorsqu'il est réalisé sur une plus longue période auprès d'une population clinique complexe et déterminer, le cas échéant, la durée optimale de l'entrainement cognitif.

Enfin, les résultats de certaines études quant aux impacts des jeux vidéo sur le système de récompense soulèvent des questions quant à l'utilisation de l'entrainement cognitif dans le traitement du TDA/H. En effet, l'utilisation des jeux vidéo est associée à une hypertrophie et suractivation du striatum, soit une structure cérébrale impliquée dans la formation des habitudes (West et al., 2015). L'entrainement cognitif tel qu'offert par le programme informatisé Cogmed pourrait améliorer la mémoire de travail ou l'attention, certes, mais aussi encourager l'utilisation de réponses automatiques soutenues par le système de récompense. Or, si les effets des jeux vidéo sur le système de récompense sont relativement bien documentés, ils demeurent peu connus en ce qui a trait à l'entrainement cognitif. 


\section{Conclusion}

Le TDA/H est un trouble neurodéveloppemental fréquent qui entraine des difficultés d'adaptation persistantes sur les plans scolaire, social et/ou familial. De par la nature complexe et hétérogène du TDA/H, sa prise en charge doit s'inscrire au sein d'une approche multimodale et être réalisée en concertation avec la personne atteinte, son entourage et les différents professionnels. En effet, la médication ne semble pas suffisante à elle seule pour permettre une véritable amélioration du fonctionnement cognitif, d'où l'importance de développer des traitements complémentaires ciblant directement les déficits neuropsychologiques associés au TDA/H (Qian et al., 2017). Les composantes neurobiologiques et cognitives du TDA/H font de l'entrainement cognitif une approche complémentaire de choix dans le traitement de ce trouble. L'entrainement de la MdT par le programme informatisé Cogmed est souvent proposé en ce sens. Le programme Cogmed s'avère actuellement le plus utilisé et suscite un grand intérêt auprès des chercheurs. Or, le maintien des effets de ce type d'intervention à plus long terme soulève encore plusieurs questions. En effet, le suivi au long cours des effets du programme Cogmed auprès d'une population TDA/H demeure peu étudié. Les résultats des études existantes quant au maintien des acquis du programme Cogmed sont mitigés, voire contradictoires (Dentz et al., 2016). De plus, les études ayant examiné les effets à long terme font généralement face à plusieurs limites sur le plan méthodologique compromettant la portée des résultats. En outre, très peu d'études contrôlent la présentation du TDA/H, la présence de comorbidités et la prise de médication, alors que ces variables sont fortement susceptibles d'interférer avec l'entrainement cognitif. De nouvelles études incluant un groupe contrôle actif, un devis en double aveugle et des mesures à plus long terme sont jugées nécessaires pour objectiver les effets du programme Cogmed et en justifier la commercialisation. 
Compte-tenu de l'absence de consensus à l'égard du maintien des acquis et des limites méthodologiques soulevées précédemment, la présente étude, en continuité avec celle de Dentz et al. (soumis), visait à suivre l'évolution des effets directs et indirects du programme Cogmed au long cours tout en tenant compte de certaines caractéristiques des participants (présentation mixte du TDA/H, troubles associés, médication). Rappelons toutefois que les résultats de l'étude de Dentz et al. (soumis) ne montraient aucun impact post-intervention sur la MdT et les diverses mesures de transfert : attention, inhibition, raisonnement non verbal, fonctionnement exécutif quotidien, symptômes liés au TDA/H, compréhension de lecture et raisonnement mathématique. Ainsi, plutôt que d'examiner le maintien des acquis, l'étude actuelle visait à examiner un possible effet à retardement du programme Cogmed, tel qu'observé dans certaines études. Les résultats n'indiquent aucune amélioration tardive six mois post-intervention. Le passage du temps ne semble pas favoriser l'apparition d'effets positifs différés sur les mesures objectives de MdT, ni sur les autres fonctions cognitives ciblées, les symptômes associés au TDA/H et le rendement scolaire. Les participants ayant pris part au programme Cogmed n'améliorent pas davantage leurs capacités d'attention, d'inhibition, de raisonnement non verbal, de compréhension de lecture et de raisonnement mathématique au cours des six mois qui suivent l'entrainement lorsqu'on les compare aux participants du groupe contrôle. De leur côté, les parents ne perçoivent pas de différence significative chez leur enfant en ce qui a trait à leurs symptômes comportementaux liés au TDA/H ainsi que l'ensemble de leur fonctionnement exécutif au quotidien. Les résultats de la présente étude ne permettent donc pas de soutenir l'efficacité au long cours du programme Cogmed auprès de jeunes ayant un TDA/H mixte avec comorbidités lorsque cette intervention est réalisée en concomitance avec un traitement pharmacologique. Malgré la commercialisation qui en est faite, nous ne pouvons recommander 
cette intervention comme traitement complémentaire à la médication dans la prise en charge du TDA/H, du moins auprès d'un échantillon spécifique comme celui ci-présent.

La médication à elle seule pourrait avoir un impact favorable sur la MdT (Holmes et al., 2010) et ainsi limiter l'impact de l'entrainement cognitif. De plus, malgré leur diagnostic de TDA/H, les participants ne présentent pas systématiquement un déficit de la MdT avant d'entreprendre le programme Cogmed, alors que cette intervention cible spécifiquement cette fonction. Ce constat remet donc en question la pertinence d'une telle approche lorsqu'aucun déficit de la MdT n'est mis en évidence préalablement à l'entrainement cognitif ou encore lorsque la médication permet de normaliser la MdT. L'objectivation d'un déficit de la MdT nous semble un prérequis important dans le cadre d'une approche combinant la médication au programme Cogmed, et ce, afin d'en maximiser les effets.

Par ailleurs, le programme Cogmed pourrait bénéficier de certains ajustements en vue d'améliorer ses effets. À cet effet, Dentz et al. (2016) proposaient une première piste qui consistait à cibler davantage l'administrateur central afin d'accroitre les impacts de l'intervention sur la MdT et favoriser la généralisation des acquis. En effet, peu d'exercices proposés par le programme Cogmed sollicitent l'administrateur central alors qu'il s'agit de la composante de la MdT la plus problématique dans le contexte d'un TDA/H (Rapport et al., 2013). L'ajout d'exercices ciblant l'administrateur central au sein du programme pourrait potentiellement favoriser la généralisation des effets, notamment sur le fonctionnement exécutif, les symptômes liés au TDA/H et les performances scolaires. Une seconde piste proposée par Dentz et al. (2016) consistait à miser sur les programmes d'entrainement cognitif ciblant un plus large éventail de fonctions cognitives comme les programmes Captain's Log et ACTIVATE. Cette approche, qui reflèterait davantage l'hétérogénéité des profils cognitifs associés au TDA/H, pourrait permettre 
une prise en charge individualisée tenant compte des déficits cognitifs spécifiques à chaque enfant. L'aspect ludique et réel du programme pourrait également être amélioré dans le but d'accroître la motivation des jeunes et favoriser leur engagement envers l'intervention. En effet, le programme Cogmed semble peu apprécié des jeunes d'un point de vue technologique. Rappelons que l'attrait à la nouveauté fait généralement partie du tempérament des individus présentant un TDA/H, de sorte qu'ils ont tendance à perdre leur intérêt plus rapidement. Différentes thématiques adaptées à l'âge des participants pourraient être proposées et sélectionnées selon les préférences du jeune. Une diversification des exercices proposés à chaque séance de même qu'un certain degré de liberté dans le choix des activités pourraient également être envisagés. Ce genre d'aménagements auraient sans doute un impact positif sur l'autodétermination des jeunes et sur leur niveau de motivation. Il serait également pertinent d'augmenter la valeur écologique du programme, c'est-à-dire la correspondance entre les exercices proposés et les activités de la vie quotidienne, afin de favoriser un meilleur transfert des apprentissages en contexte réel (Cicerone et al., 2011; Qian et al., 2017).

Finalement, une dernière piste pourrait consister à modifier certaines modalités associées à l'entrainement cognitif, notamment en ce qui a trait au temps d'entrainement par séance, au nombre de répétitions et à la durée totale de l'entrainement. L'augmentation de l'intensité de l'entrainement cognitif en termes de durée et/ou de niveau de difficulté pourrait être particulièrement pertinente auprès des jeunes présentant un TDA/H avec comorbidités (Shinaver et al., 2014). La sévérité du tableau clinique et des déficits associés pourrait en effet moduler le degré de neuroplasticité et limiter l'efficacité de l'entrainement cognitif (Chacko et al., 2013). Un entrainement prolongé pourrait davantage correspondre aux besoins thérapeutiques complexes de ces jeunes et ainsi favoriser les gains immédiats, la généralisation des effets et le 
maintien des acquis dans le temps. Une telle approche mériterait d'être investiguée dans de futures études, d'autant plus que les mécanismes actifs sous-tendant l'entrainement cognitif de même que les facteurs permettant de prédire les effets à long terme demeurent à ce jour peu connus (Shinaver et al., 2014). 


\section{Références}

Adler, L. D. et Nierenberg, A. A. (2010). Review of medication adherence in children and adults with ADHD. Postgraduate medicine, 122(1), 184-191.

Advokat, C. (2010). What are the cognitive effects of stimulant medications? Emphasis on adults with attention-deficit/hyperactivity disorder (ADHD). Neuroscience \& Biobehavioral Reviews, 34(8), 1256-1266.

Ahmadi, N., Mohammadi, M. R., Araghi, S. M. et Zarafshan, H. (2014). Neurocognitive profile of children with attention deficit hyperactivity disorders (ADHD): a comparison between subtypes. Iranian journal of psychiatry, 9(4), 197.

Alloway, T. P. et Copello, E. (2013). Working memory: The what, the why, and the how. The Australian Educational and Developmental Psychologist, 30(02), 105-118.

American Academy of Pediatrics. (2011). ADHD: Clinical practice guidelines for the diagnosis, evaluation and treatment of attention-deficit/hyperactivity disorder in children and adolescents. Pediatrics, 128(5), 1007-1022.

American Psychiatric Association (2000). Diagnostic and statistical manual of mental disorders: DSM-IV-TR (4e éd.). Washington, DC: American Psychiatric Association.

American Psychiatric Association (2013). Diagnostic and statistical manual of mental disorders DSM-5 (5e éd.). Whashington, DC: American Psychiatric Publishing.

Arnsten, A. F., \& Rubia, K. (2012). Neurobiological circuits regulating attention, cognitive control, motivation, and emotion: disruptions in neurodevelopmental psychiatric disorders. Journal of the American Academy of Child \& Adolescent Psychiatry, 51(4), 356-367.

Astle, D. E., Barnes, J. J., Baker, K., Colclough, G. L. et Woolrich, M. W. (2015). Cognitive training enhances intrinsic brain connectivity in childhood. Journal of Neuroscience, 35(16), 6277-6283.

Bäckman, L. et Nyberg, L. (2013). Dopamine and training-related working-memory improvement. Neuroscience \& Biobehavioral Reviews, 37(9), 2209-2219.

Baddeley, A. D., \& Hitch, G. (1974). Working memory. Psychology of learning and motivation, 8, 47-89.

Baddeley, A. (2012). Working memory: theories, models, and controversies. Annual review of psychology, 63, 1-29.

Barkley, R. A. (1997). Behavioral inhibition, sustained attention, and executive functions: 
constructing a unifying theory of ADHD. Psychological Bulletin, 121(1), 65-94.

Barkley, R. A. (2002). International consensus statement on ADHD. Journal of the American Academy of Child and Adolescent Psychiatry, 41(12), 1389.

Beck, S. J., Hanson, C. A., Puffenberger, S. S., Benninger, K. L. et Benninger, W. B. (2010). A controlled trial of working memory training for children and adolescents with ADHD. Journal of Clinical Child \& Adolescent Psychology, 39(6), 825-836.

Berger, I., Dor, T., Nevo, Y. et Goldzweig, G. (2008). Attitudes Toward Attention-Deficit Hyperactivity Disorder (ADHD) Treatment: Parents' and Children's Perspectives. Journal of Child Neurology, 23(9), 1036-1042.

Biederman, J., Monuteaux, M. C., Mick, E., Spencer, T., Wilens, T. E., Silva, J. M...Faraone, S. V. (2006). Young adult outcome of attention deficit hyperactivity disorder: a controlled 10-year follow-up study. Psychological Medecine, 36(2), 167-179.

Biederman, J., Petty, C. R., Woodworth, K. Y., Lomedico, A., Hyder, L. L. et Faraone, S. V. (2012). Adult outcome of attention-deficit/hyperactivity disorder: a controlled 16-year follow-up study. The Journal of Clinical Psychiatry, 73(7), 941-950. doi: 10.4088/JCP. $11 \mathrm{~m} 07529$

Bigorra, A., Garolera, M., Guijarro, S. et Hervás, A. (2016). Long-term far-transfer effects of working memory training in children with ADHD: a randomized controlled trial. European child \& adolescent psychiatry, 25(8), 853-867.

Bolfer, C., Pacheco, S. P., Tsunemi, M. H., Carreira, W. S., Casella, B. B., \& Casella, E. B. (2017). Attention-deficit/hyperactivity disorder: the impact of methylphenidate on working memory, inhibition capacity and mental flexibility. Arquivos de NeuroPsiquiatria, 75(4), 204-208.

Brehmer, Y., Westerberg, H. et Bäckman, L. (2012). Working-memory training in younger and older adults: training gains, transfer, and maintenance. Frontiers in human neuroscience, 6 .

Burgess, G. C., Depue, B. E., Ruzic, L.,Willcutt, E. G., Du, Y. P. et Banich, M. T. (2010). Attentional control activation relates to working memory in attentiondeficit/hyperactivity disorder. Biological Psychiatry, 67, 632-640.

Chacko, A., Bedard, A. C., Marks, D. J., Feirsen, N., Uderman, J. Z., Chimiklis, A ... Ramon, M. (2014). A randomized clinical trial of Cogmed working memory training in schoolage children with ADHD: a replication in a diverse sample using a control condition. Journal of Child Psychology and Psychiatry, 55(3), 247-255.

Chacko, A., Feirsen, N., Bedard, A. C., Marks, D., Uderman, J. Z. et Chimiklis, A. (2013). Cogmed working memory training for youth with ADHD: a closer examination of 
efficacy utilizing evidence-based criteria. Journal of Clinical Child \& Adolescent Psychology, 42(6), 769-783.

Conners, C. K. (2000). Conners' Continuous Performance Test II, Multi-Health Systems Inc.

Conners, C. K. (2008). Conners, $3^{e}$ édition. Multi-Health Systems Inc.

Conners, C. K. et Sitarenios, G. (2011). Conners' continuous performance test (CPT). In Encyclopedia of clinical neuropsychology (pp. 681-683). Springer New York.

Cortese, S., Ferrin, M., Brandeis, D., Buitelaar, J., Daley, D., Dittmann, R. W... Zuddas, A. (2015). Cognitive training for attention-deficit/hyperactivity disorder: meta-analysis of clinical and neuropsychological outcomes from randomized controlled trials. Journal of the American Academy of Child \& Adolescent Psychiatry, 54(3), 164174.

Cortese, S., Holtmann, M., Banaschewski, T., Buitelaar, J., Coghill, D., Danckaerts, M... Sergeant, J. (2013). Practitioner review: current best practice in the management of adverse events during treatment with ADHD medications in children and adolescents. Journal of Child Psychology and Psychiatry, 54(3), 227-246.

Cortese, S., Kelly, C., Chabernaud, C., Proal, E., Di Martino, A., Milham, M. P. et Castellanos, F. X. (2012). Toward systems neuroscience of ADHD: A meta-analysis of 55 fMRI studies. The American Journal of Psychiatry, 169(10), 1038-1055.

Deforge, H., (2011). Prise en charge des troubles attentionnels et exécutifs chez l'enfant - La remédiation cognitive : pratiques et perspectives. Développements, 5-20.

Dentz, A., Guay, M.-C., Gauthier, B., Romo, L. et Parent, V. (soumis). Is the Cogmed program effective for youths with ADHD under pharmacological treatment? Applied Cognitive Psychology.

Dentz, A., Parent, V., Gauthier, B., Guay, M. C. et Romo, L. (2016). L'entraînement de la mémoire de travail par le programme Cogmed et le TDAH. Psychologie Française, 61(2), 139-151.

Dickstein, S. G., Bannon, K., Xavier Castellanos, F. et Milham, M. P. (2006). The neural correlates of attention deficit hyperactivity disorder: An ALE meta-analysis. Journal of Child Psychology and Psychiatry, 47(10), 1051-1062.

Dovis, S., Oord, S. V., der Wiers, R. W. et Prins, P. J. M. (2013). What part of working memory is not working in ADHD? Short-term memory, the central executive and effects of reinforcement. Journal of Abnormal Child Psychology, 41(6), 901-917.

Dongen-Boomsma, M., Vollebregt, M. A., Buitelaar, J. K. et Slaats-Willemse, D. (2014). Working memory training in young children with ADHD: a randomized placebo- 
controlled trial. Journal of Child Psychology and Psychiatry, 55(8), 886-896.

Dunning, D. L., Holmes, J. et Gathercole, S. E. (2013). Does working memory training lead to generalized improvements in children with low working memory? A randomized controlled trial. Developmental Science, 16(6), 915-925.

Durston, S., van Belle, J. et Zeeuw, P. (2011). Differentiating Frontostriatal and FrontoCerebellar Circuits in Attention-Deficit/Hyperactivity Disorder. Biological Psychiatry, 69, 1178-1184.

Egeland, J., Aarlien, A. K. et Saunes, B. K. (2013). Few effects of far transfer of working memory training in ADHD: a randomized controlled trial. PloS one, 8(10), e75660.

Elia, J., Ambrosini, P. et Berrettini, W. (2008). ADHD characteristics: I. Concurrent comorbidity patterns in children \& adolescents. Child and adolescent psychiatry and mental health, 2(1), 15.

Faraone, S. V., Biederman, J., Spencer, T. J. et Aleardi, M. (2006). Comparing the Efficacy of Medications for ADHD Using Meta-analysis. Medscape General Medicine. 8(4).

Faraone, S. V., \& Buitelaar, J. (2010). Comparing the efficacy of stimulants for ADHD in children and adolescents using meta-analysis. European child \& adolescent psychiatry, 19(4), 353-364.

Farias, A. C., Cordeiro, M. L., Felden, E. P., Bara, T. S., Benko, C. R., Coutinho, D., ... \& McCracken, J. T. (2017). attention-memory training yields behavioral and academic improvements in children diagnosed with attention-deficit hyperactivity disorder comorbid with a learning disorder. Neuropsychiatric Disease and Treatment, 13, 1761.

Friedman, L. A., \& Rapoport, J. L. (2015). Brain development in ADHD. Current opinion in neurobiology, 30, 106-111.

Fuermaier, A. B., Tucha, L., Koerts, J., Weisbrod, M., Lange, K. W., Aschenbrenner, S. et Tucha, O. (2017). Effects of methylphenidate on memory functions of adults with ADHD. Applied Neuropsychology: Adult, 24(3), 199-211.

Gathercole, S. E. (2014). Commentary: Working memory training and ADHD-where does its potential lie? Reflections on Chacko et al. (2014). Journal of Child Psychology and Psychiatry, 55(3), 256-257.

Gibson, B. S., Gondoli, D. M., Johnson, A. C., Steeger, C. M., Dobrzenski, B. A. et Morrissey, R. A. (2011). Component analysis of verbal versus spatial working memory training in adolescents with ADHD: A randomized, controlled trial. Child Neuropsychology, 17(6), 546-563.

Gioia, G., Isquith, P., Guy, S. C. et Kenworth, L. (2000). Behavior Rating of Inventory of 
Executive Function: Professional Manual. Professional Manual Lutz, FL: Psychological Assessment Resources.

Giroux, S., Parent, V. et Guay, M. C. (2010). La remédiation cognitive et la remédiation métacognitive pour les personnes ayant un TDAH : deux stratégies d'intervention novatrices et pourquoi pas complémentaires?. Journal de thérapie comportementale et cognitive, 20(3), 87-92.

Graham, J., Banaschewski, T., Buitelaar, J., Coghill, D., Danckaerts, M., Dittmann, R. W., ... \& Hulpke-Wette, M. (2011). European guidelines on managing adverse effects of medication for ADHD. European child \& adolescent psychiatry, 20(1), 17-37.

Graham, A. R., \& Benninger, W. B. (2016). Parental perceptions of the efficacy of Cogmed working memory training. Applied Neuropsychology: Child, 5(3), 173-179.

Graham, J. et Coghill, D. (2008). Adverse effects of pharmacotherapies for attention-deficit hyperactivity disorder. CNS drugs, 22(3), 213-237.

Gray, S. A., Chaban, P., Martinussen, R., Goldberg, R., Gotlieb, H., Kronitz, R... Tannock, R. (2012). Effects of a computerized working memory training program on working memory, attention, and academics in adolescents with severe LD and comorbid ADHD: a randomized controlled trial. Journal of Child Psychology and Psychiatry, 53(12), 1277-1284.

Green, C. T., Long, D. L., Green, D., Iosif, A. M., Dixon, J. F., Miller, M. R ... Schweitzer, J. B. (2012). Will working memory training generalize to improve off-task behavior in children with attention-deficit/hyperactivity disorder?. Neurotherapeutics, 9(3), 639648.

Gropper, R. J., Gotlieb, H., Kronitz, R. et Tannock, R. (2014). Working memory training in college students with ADHD or LD. Journal of Attention Disorders, 18(4), 331-345.

Holmes, J., Gathercole, S. E. et Dunning, D. L. (2009). Adaptive training leads to sustained enhancement of poor working memory in children. Developmental science, 12(4).

Holmes, J., Gathercole, S. E., Place, M., Dunning, D. L., Hilton, K. A. et Elliott, J. G. (2010). Working memory deficits can be overcome: Impacts of training and medication on working memory in children with ADHD. Applied Cognitive Psychology, 24(6), 827836.

Hovik, K. T., Saunes, B. K., Aarlien, A. K. et Egeland, J. (2013). RCT of working memory training in ADHD: long-term near-transfer effects. PLoS One, 8(12), e80561.

Kao, G. S. et Thomas, H. M. (2010). Test Review: C. Keith Conners Conners 3rd Edition Toronto, Ontario, Canada: Multi-Health Systems, 2008. Journal of Psychoeducational Assessment, 28(6), 598-602. 
Karalunas, S. L., Gustafsson, H. C., Dieckmann, N. F., Tipsord, J., Mitchell, S. H., \& Nigg, J. T. (2017). Heterogeneity in development of aspects of working memory predicts longitudinal attention deficit hyperactivity disorder symptom change. Journal of abnormal psychology, 126(6), 774.

Kasper, L. J., Alderson, R. M. et Hudec, K. L. (2012). Moderators of working memory deficits in children with attention-deficit/hyperactivity disorder (ADHD): a meta-analytic review. Clinical psychology review, 32(7), 605-617.

Klingberg, T. (2010). Training and plasticity of working memory. Trends in Cognitive Sciences, 14(7), 317-324.

Klingberg, T., Fernell, E., Olesen, P. J., Johnson, M., Gustafsson, P., Dahlström, K... Westerberg, H. (2005). Computerized training of working memory in children with ADHD - a randomized, controlled trial. Journal of the American Academy of Child et Adolescent Psychiatry, 44(2), 177-186.

Klingberg, T., Forssberg, H. et Westerberg, H. (2002). Training of working memory in children with ADHD. Journal of Clinical and Experimental Neuropsychology, 24(6), 781-791.

Kofler, M. J., Rapport, M.D., Bolden, J., Sarver, D. E., Raiker, J. S. et Alderson, R. M. (2011). Working memory deficits and social problems in children with ADHD. Journal of Abnormal Child Psychology, 39(6), 805-817. doi : 10.1007/s10802-011-9492-8

Larson, K., Russ, S. A., Kahn, R. S. et Halfon, N. (2011). Patterns of comorbidity, functioning, and service use for US children with ADHD, 2007. Pediatrics, peds2010 .

Mawjee, K., Woltering, S., \& Tannock, R. (2015). Working memory training in postsecondary students with ADHD: A randomized controlled study. PloS one, 10(9), e0137173.

McGurk, S. R., Twamley, E. W., Sitzer, D. I., McHugo, G. J. et Mueser, K. T. (2007). A meta-analysis of cognitive remediation in schizophrenia. American Journal of Psychiatry, 164(12), 1791-1802.

McNab, F., Varrone, A., Farde, L., Jucaite, A., Bystritsky, P., Forssberg, H. et Klingberg, T. (2009). Changes in cortical dopamine D1 receptor binding associated with cognitive training. Science, 323(5915), 800-802.

Melby-Lervåg, M. et Hulme, C. (2013). Is working memory training effective? A meta- analytic review. Developmental psychology, 49(2), 270. 
Mezzacappa, E. et Buckner, J. C. (2010). Working memory training for children with attention problems or hyperactivity: A school-based pilot study. School Mental Health, 2(4), 202-208.

Miller, M., Nevado-Montenegro, A. J. et Hinshaw, S. P. (2012). Childhood executive function continues to predict outcomes in young adult females with and without childhooddiagnosed ADHD. Journal of abnormal child psychology, 40(5), 657-668.

Molina, B., Hinshaw, S. P., Swanson, J.M., Arnold, L. E., Vitiello, B., Jensen, P.S...Houck, P. R. (2009). The MTA at 8 years: Prospective follow-up of children treated for combined-type ADHD in a multisite study. Journal of the American Academy of Child and Adolescent Psychiatry, 48(5), 484-500.

Nigg, J. T., Willcutt, E. G., Doyle, A. E. et Sonuga-Barke, E. J. S. (2005). Causal heterogeneity in attention-deficit/hyperactivity disorder: Do we need neuropsychologically impaired subtypes? Biological Psychiatry, 57(11), 1224-1230.

Olesen, P. J., Westerberg, H. et Klingberg, T. (2004). Increased prefrontal and parietal activity after training of working memory. Nature neuroscience, 7(1), 75-79.

Palladino, P. et Ferrari, M. (2013). Interference control in working memory: Comparing groups of children with atypical development. Child Neuropsychology, 19(1), 37-54.

Polanczyk, G. V., Willcutt, E. G., Salum, G. A., Kieling, C. et Rohde, L. A. (2014). ADHD prevalence estimates across three decades: an updated systematic review and metaregression analysis. International journal of epidemiology, 43(2), 434-442.

Qian, Y., Chen, M., Shuai, L., Cao, Q. J., Yang, L. et Wang, Y. F. (2017). Effect of an Ecological Executive Skill Training Program for School-aged Children with Attention Deficit Hyperactivity Disorder: A Randomized Controlled Clinical Trial. Chinese Medical Journal, 130(13), 1513.

Raiker, J. S., Rapport, M. D., Kofler, M. J. et Sarver, D. E. (2012). Objectively-measured impulsivity and attention-deficit/hyperactivity disorder (ADHD): testing competing predictions from the working memory and behavioral inhibition models of ADHD.

Journal of Abnormal Child Psychology, 40(5), 699-713.

Rapport, M. D., Alderson, R. M., Kofler, M. J., Sarver, D. E., Bolden, J. et Sims, V. (2008). Working memory deficits in boys with attention-deficit/hyperactivity disorder (ADHD): the contribution of central executive and subsystem processes. Journal of abnormal child psychology, 36(6), 825-837.

Rapport, M. D., Bolden, J., Kofler, M. J., Sarver, D. E., Raiker, J. S. et Alderson, R. M. (2009). Hyperactivity in boys with attentiondeficit/hyperactivity disorder (ADHD): a ubiquitous core symptom or manifestation of working memory deficits? Journal of Abnormal Child Psychology, 37(4), 521-534. 
Rapport, M. D., Orban, S. A., Kofler, M. J. et Friedman, L. M. (2013). Do programs designed to train working memory, other executive functions, and attention benefit children with ADHD? A meta-analytic review of cognitive, academic, and behavioral outcomes. Clinical psychology review, 33(8), 1237-1252.

Raven, J. C., Court, J. H. et Raven, J. (1998). Progressive Matrices Couleurs. Paris: Les Editions du Centre de Psychologie Appliquée (ECPA).

Rizzo, R., Gulisano, M., Calì, P. V. et Curatolo, P. (2013). Tourette syndrome and comorbid ADHD: current pharmacological treatment options. European journal of paediatric neurology, 17(5), 421-428.

Rogers, M., Hwang, H., Toplak, M., Weiss, M. et Tannock, R. (2011). Inattention, working memory, and academic achievement in adolescents referred for attentiondeficit/hyperactivity disorder (ADHD). Child Neuropsychology: A Journal on Normal and Abnormal Development in Childhood and Adolescence, 17(5), 444-458.

Rosa, V. D. O., Schmitz, M., Moreira-Maia, C. R., Wagner, F., Londero, I., Bassotto, C. D. F., ... Rohde, L. A. P. (2017). Computerized cognitive training in children and adolescents with attention deficit/hyperactivity disorder as add-on treatment to stimulants: feasibility study and protocol description. Trends in psychiatry and psychotherapy, 39(2), 65-76.

Rutledge, K. J., van den Bos, W., McClure, S. M. et Schweitzer, J. B. (2012). Training Cognition in ADHD: Current Findings, Borrowed Concepts, and Future Directions. Neurotherapeutics, 9(3), 542-558.

Safren, S. A., Sprich, S., Mimiaga, M. J., Surman, C., Knouse, L., Groves, M. et Otto, M. W. (2010). Cognitive behavioral therapy vs relaxation with educational support for medication-treated adults with ADHD and persistent symptoms: a randomized controlled trial. Jama, 304(8), 875-880.

Schwaighofer, M., Fischer, F. et Bühner, M. (2015). Does working memory training transfer? A meta-analysis including training conditions as moderators. Educational Psychologist, 50(2), 138-166.

Seixas, M., Weiss, M. et Müller, U. (2012). Systematic review of national and international guidelines on attention-deficit hyperactivity disorder. Journal of Psychopharmacology, 26(6), 753-765.

Shaw, P., Eckstrand, K., Sharp, W., Blumenthal, J., Lerch, J. P., Greenstein, D...Rapport, J. L. (2007). Attention-deficit/hyperactivity disorder is characterized by a delay in cortical maturation. PNAS, 104(49), 19649-19654.

Shaw, P., Malek, M., Watson, B., Sharp, W., Evans, A. et Greenstein, D. (2012). Development of cortical surface area and gyrification in attention-deficit/hyperactivity disorder. 
Biological Psychiatry, 72(3), 191-197.

Shinaver III, C. S., Entwistle, P. C. et Söderqvist, S. (2014). Cogmed WM training: reviewing the reviews. Applied Neuropsychology: Child, 3(3), 163-172.

Shipstead, Z., Redick, T. S. et Engle, R. W. (2012). Is working memory training effective?. Psychological bulletin, 138(4), 628.

Sjöwall, D., Roth, L., Lindqvist, S. et Thorell, L. B. (2013). Multiple deficits in ADHD: Executive dysfunction, delay aversion, reaction time variability and emotional deficits. Journal of Child Psychology and Psychiatry, 54(6), 619-627.

Sjöwall, D. et Thorell, L. B. (2014). Functional impairments in attention-deficit hyperactivity disorder: The mediating role of neuropsychological functioning. Developmental Neuropsychology, 39(3), 187-204.

Söderqvist, S. et Nutley, S. (2015). Cogmed working memory training: claims and evidence complete version $\left(3^{\mathrm{e}} \quad\right.$ éd.). Pearson Clincal Assessment. Repéré à http://www.cogmed.com/research

Söderqvist, S. et Nutley, S. B. (2015). Working memory training is associated with long term attainments in math and reading. Frontiers in psychology, 6.

Sohlberg, M. M. et Mateer, C. A. (2017). Cognitive rehabilitation: An integrative neuropsychological approach. Guilford Publications.

Sonuga-Barke, E. J. S. (2002). Psychological Heterogeneity in ADHD: a dual pathway model of behaviour and cognition. Behavioural Brain Research, 130, 29-36.

Sonuga-Barke, E. J. S. (2003). The dual pathway model of AD/HD: an elaboration of neurodevelopmental characteristics. Neuroscience and Biobehavioral Reviews, 27, 593-604.

Sonuga-Barke, E. J. S. (2005). Causal Models of Attention-Deficit/Hyperactivity Disorder: From Common Simple Deficits to Multiple Developmental Pathways. Biological Psychiatry, 57(11), 1231-1238.

Sonuga-Barke, E. J. S., Bitsakou, P. et Thompson, M. (2010). Beyond the Dual Pathway Model: Evidence for the Dissociation of Timing, Inhibitory, and Delay-Related Impairments in Attention-Deficit/Hyperactivity Disorder. Journal of The American Academy of Child and Adolescent Psychiatry, 49(4), 345-355.

Sonuga-Barke, E. J., Brandeis, D., Cortese, S., Daley, D., Ferrin, M., Holtmann, M... Dittmann, R. W. (2013). Nonpharmacological interventions for ADHD: systematic review and meta-analyses of randomized controlled trials of dietary and psychological treatments. American Journal of Psychiatry, 170(3), 275-289. 
Sonuga-Barke, E. J. et Halperin, J. M. (2010). Developmental phenotypes and causal pathways in attention deficit/hyperactivity disorder: potential targets for early intervention?. Journal of Child Psychology and Psychiatry, 51(4), 368-389.

Spencer-Smith, M. et Klingberg, T. (2015). Benefits of a working memory training program for inattention in daily life: a systematic review and meta-analysis. PLoS One, 10(3), e0119522.

Swanson, J., Baler, R. D. et Volkow, N. D. (2011). Understanding the effects of stimulant medications on cognition in individuals with attention-deficit hyperactivity disorder: a decade of progress. Neuropsychopharmacology, 36(1), 207-226.

Stevens, M. C., Gaynor, A., Bessette, K. L. et Pearlson, G. D. (2016). A preliminary study of the effects of working memory training on brain function. Brain imaging and behavior, 10(2), 387-407.

Strand, M. T., Hawk, L. W., Bubnik, M., Shiels, K., Pelham, W. E. et Waxmonsky, J. G. (2012). Improving working memory in children with attention-deficit/hyperactivity disorder: the separate and combined effects of incentives and stimulant medication. Journal of abnormal child psychology, 40(7), 1193-1207.

Swanson, L. et Kim, K. (2007). Working memory, short-term memory, and naming speed as predictors of children's mathematical performance. Intelligence, 35(2), 151-168.

Tabachnick, B. G. et Fidell, L. S. (2007). Using Multivariate Statistics. Pearson Education, Limited.

Thompson, T. W., Waskom, M. L. et Gabrieli, J. D. (2016). Intensive working memory training produces functional changes in large-scale frontoparietal networks. Journal of cognitive neuroscience.

Valera, E. M., Faraone, S. V., Murray, K. E. et Seidman, L. J. (2007). Meta-analysis of structural imaging findings in attention-deficit/hyperactivity disorder. Biological psychiatry, 61(12), 1361-1369.

Van der Donk, M., Hiemstra-Beernink, A. C., Tjeenk-Kalff, A., Van Der Leij, A. et Lindauer, R. (2015). Cognitive training for children with ADHD: a randomized controlled trial of cogmed working memory training and 'paying attention in class'. Frontiers in psychology, 6, 1081.

Van der Donk, M. L., Hiemstra-Beernink, A. C., Tjeenk-Kalff, A. C., van der Leij, A. et Lindauer, R. J. (2016). Predictors and moderators of treatment outcome in cognitive training for children with ADHD. Journal of attention disorders, 1087054716632876.

Van der Molen, M., Van Luit, J. E. H., Van der Molen, M. W., Klugkist, I., \& Jongmans, M. J. (2010). Effectiveness of a computerised working memory training in adolescents with 
mild to borderline intellectual disabilities. Journal of Intellectual Disability Research, 54(5), 433-447.

Van der Oord, S., Prins, P. J., Oosterlaan, J. et Emmelkamp, P. M. (2008). Efficacy of methylphenidate, psychosocial treatments and their combination in school-aged children with ADHD: a meta-analysis. Clinical psychology review, 28(5), 783-800.

Van Hulst, B. M., de Zeeuw, P. et Durston, S. (2015). Distinct neuropsychological profiles within ADHD: a latent class analysis of cognitive control, reward sensitivity and timing. Psychological Medicine, 45(04), 735-745.

Vinogradov, S., Fisher, M. et de Villers-Sidani, E. (2012). Cognitive training for impaired neural systems in neuropsychiatric illness. Neuropsychopharmacology Reviews, 37, 43-76.

Wechsler, D. (2005a). Échelle d'intelligence pour enfants de Wechsler, 4e édition pour francophones du Canada. Toronto : Harcourt Assessment Inc.

Wechsler, D. (2005b). Test de rendement individuel de Wechsler, 4e édition, version pour francophones du Canada. Toronto : Harcourt Assessment Inc.

Wechsler, D. (2006). Échelle non verbale d'aptitude de Wechsler, version canadienne. Toronto : Harcourt Assessment Inc.

Wehmeier, P. M., Schacht, A. et Barkley, R. A. (2010). Social and emotional impairment in children and adolescents with ADHD and the impact on quality of life. Journal of Adolescent Health, 46(3), 209-217.

West, G. L., Drisdelle, B. L., Konishi, K., Jackson, J., Jolicoeur, P. et Bohbot, V. D. (2015). Habitual action video game playing is associated with caudate nucleus-dependent navigational strategies. In Proc. R. Soc. B (Vol. 282, No. 1808, p. 20142952). The Royal Society.

Westerberg, H. et Klingberg, T. (2007). Changes in cortical activity after training of working memory - a single-subject analysis. Physiology and Behavior, 92(1-2), 186-192.

Willcutt, E. G., Doyle, A. E., Nigg, J. T., Faraone, S. V. et Pennington, B. F. (2005). Validity of the executive function theory of attention-deficit/hyperactivity disorder: a metaanalytic review. Biological psychiatry, 57(11), 1336-1346.

Yang, T. X., Allen, R. J. et Gathercole, S. E. (2016). Examining the role of working memory resources in following spoken instructions. Journal of Cognitive Psychology, 28(2), 186198. 